\title{
Entire surfaces of constant curvature in Minkowski 3-space
}

\author{
Francesco Bonsante ${ }^{1}(\mathbb{1}) \cdot$ Andrea Seppi $^{2} \cdot$ Peter Smillie $^{3}([)$
}

Received: 29 May 2018 / Published online: 23 March 2019

(c) The Author(s) 2019

\begin{abstract}
This paper concerns the global theory of properly embedded spacelike surfaces in three-dimensional Minkowski space in relation to their Gaussian curvature. We prove that every regular domain which is not a wedge is uniquely foliated by properly embedded convex surfaces of constant Gaussian curvature. This is a consequence of our classification of surfaces with bounded prescribed Gaussian curvature, sometimes called the Minkowski problem, for which partial results were obtained by Li, GuanJian-Schoen, and Bonsante-Seppi. Some applications to minimal Lagrangian selfmaps of the hyperbolic plane are obtained.
\end{abstract}

Mathematics Subject Classification Primary 53C42; Secondary 35J96 · 53B30 . $53 \mathrm{C} 50$

Communicated by F. C. Marques.

The first author was partially supported by Blue Sky Research project "Analytic and geometric properties of low-dimensional manifolds". The first two authors are members of the national research group GNSAGA.

$凶 \quad$ Peter Smillie

smillie@caltech.edu

Francesco Bonsante

bonfra07@unipv.it

Andrea Seppi

andrea.seppi@univ-grenoble-alpes.fr

1 Dipartimento di Matematica "Felice Casorati”, Università degli Studi di Pavia, Via Ferrata 5, 27100 Pavia, Italy

2 CNRS and Université Grenoble Alpes, 100 Rue des Mathématiques, 38610 Gières, France

3 Division of Physics, Mathematics and Astronomy, Caltech, Mail Code 253-37, Pasadena, CA 91125 , USA 


\section{Introduction}

Minkowski 3-space is the simply connected geodesically complete flat Lorentzian manifold $\mathbb{R}^{2,1}=\left(\mathbb{R}^{3}, d x_{1}^{2}+d x_{2}^{2}-d x_{3}^{2}\right)$. A $C^{1}$ immersed surface $\Sigma$ in $\mathbb{R}^{2,1}$ is called spacelike if the restriction of the Lorentzian metric to $T \Sigma$ is a Riemannian metric. Any spacelike surface is locally a graph of the form $x_{3}=f\left(x_{1}, x_{2}\right)$ for some function $f \in C^{1}\left(\mathbb{R}^{2}\right)$ which is strictly 1-Lipschitz with respect to the Euclidean metric of the plane. The aim of the paper is to provide a full classification of properly embedded spacelike surfaces with constant Gaussian curvature (CGC) in Minkowski space in terms of their asymptotic behavior.

Let us explain more precisely the content of the classification. Given a properly embedded spacelike surface $\Sigma$ in $\mathbb{R}^{2,1}$, its domain of dependence $\mathcal{D}_{\Sigma}$ is the region of $\mathbb{R}^{2,1}$ consisting of those points through which any inextendable causal path must meet $\Sigma$ (Definition 1.13). We show in Corollary 1.17 that the domain of dependence of a properly embedded CGC surface $\Sigma$ is a regular domain up to time-reversal. Here the terminology is taken from [3]: a regular domain is a convex open domain in $\mathbb{R}^{2,1}$ that is the intersection of the future of its null support planes and is neither the whole $\mathbb{R}^{2,1}$ nor the future of a single null plane.

Among regular domains we call wedges those domains which are obtained as the intersection of the futures of exactly two null planes. It turns out that a wedge is never the domain of dependence of a properly embedded CGC surface. The main goal of this paper is to prove that aside from this case, every regular domain is the domain of dependence of exactly one properly embedded surface of constant Gaussian curvature $K$, for any fixed $K \in(0,+\infty)$. In this paper, $K$ is the extrinsic Gaussian curvature, which is the determinant of the shape operator and the negative of the intrinsic Gaussian curvature.

Theorem A Fix $K>0$. Given any regular domain $\mathcal{D} \subset \mathbb{R}^{2,1}$ which is not a wedge, there exists a unique properly embedded $C G C-K$ surface whose domain of dependence is $\mathcal{D}$.

Once we conclude as a consequence of Corollary 1.17 that the domain of dependence of every future-convex CGC- $K$ surface is a regular domain and not a wedge, we immediately have the corollary:

Corollary B Fix $K>0$. There is a bijection from the set of properly embedded futureconvex $C G C K$-surfaces in $\mathbb{R}^{2,1}$ to the set of regular domains which are not a wedge, given by associating to a surface its domain of dependence.

We may restate our main theorem in terms of lower semi-continuous functions on the circle. In the Klein model of the hyperbolic plane $\mathbb{H}^{2}$, points on the boundary of $\mathbb{H}^{2}$ represent lightlike directions in $\mathbb{R}^{2,1}$, which by the duality induced by the Lorentzian inner product are in bijection with null linear planes. The space of null affine planes in $\mathbb{R}^{2,1}$ is naturally identified to a cylinder $\partial \mathbb{H}^{2} \times \mathbb{R}$. Two points $\partial \mathbb{H}^{2} \times \mathbb{R}$ correspond to parallel planes if and only if their first components coincide. From this point of view regular domains are in bijection with lower-semicontinuous functions $\varphi: \partial \mathbb{H}^{2} \rightarrow \mathbb{R} \cup\{+\infty\}$ that are finite at least on two points (Proposition 2.5). We 
will call $\mathcal{D}_{\varphi}$ the domain corresponding to the function $\varphi$. If $\Sigma$ is the graph of an entire convex function $f: \mathbb{R}^{2} \rightarrow \mathbb{R}$, we call $\Sigma$ entire. A simple argument (Proposition 1.10) shows that every properly immersed spacelike surface is entire. It was proved in [4, Subsection 2.3] that the function $\varphi$ corresponding to $\mathcal{D}_{\Sigma}$ is given by

$$
\varphi(\mathrm{y})=-\lim _{r \rightarrow+\infty}(f(r \mathrm{y})-r)
$$

In this way the function $\varphi$ encodes the asymptotic behavior of the surface $\Sigma$. The graph of $\varphi$ can also be regarded as the asymptotic boundary of $\Sigma$ in the Penrose causal compactification of $\mathbb{R}^{2,1}$, but this point of view will not developed here.

Therefore Theorem A establishes a correspondence between entire CGC graphs and lower semi-continuous functions on the circle which may take the value $+\infty$ :

Corollary C Fix $K>0$. There is a bijection between the set of future-convex entire surfaces of constant Gaussian curvature $K$ in $\mathbb{R}^{2,1}$ and the set of lower semicontinuous functions $\varphi: \partial \mathbb{H}^{2} \rightarrow \mathbb{R} \cup\{+\infty\}$ finite on at least three points.

Next, we will prove that any regular domain that is not a wedge is foliated by CGC surfaces with constant Gaussian curvature ranging from 0 to $\infty$ :

Theorem D For every regular domain $\mathcal{D}$ in $\mathbb{R}^{2,1}$ which is not a wedge, there exists a unique foliation by properly embedded CGC $K$-surfaces, with $K \in(0, \infty)$.

As a result, the function $\tau=K^{-1}$ gives a canonical proper function with time-like gradient on every regular domain $\mathcal{D}$. It is an example of a geometric canonical time function, called the $K$-time in [1].

The study of CGC surfaces in Minkowski space goes back at least to Hano and Nomizu [12] who first pointed out the existence of non-standard isometric immersions of $\mathbb{H}^{2}$ in $\mathbb{R}^{2,1}$. In [15] An-Min Li proved the existence part of Theorem A and Corollary $\mathrm{C}$ in the case that $\varphi$ is smooth. This result was improved by Guan, Jian and Schoen [9]: they proved the existence of an entire CGC $K$-surface only assuming $\varphi$ is Lipschitz and possibly infinite on a single open arc. In another direction, Barbot, Béguin and Zeghib proved in [1] that any regular domain invariant by an affine deformation of a uniform lattice in $\mathrm{SO}(2,1)$ contains a CGC $K$-surface. In [4] the first two authors proved the existence of a CGC surface in a given regular domain under the assumption that the corresponding function $\varphi$ is lower semi-continuous and bounded. Moreover in that work it was proved that entire CGC surfaces with bounded second fundamental form are in bijection with regular domains whose corresponding function is Zygmund continuous.

In higher dimensions the problem can be generalized in different ways. Li's original theorem applies to hypersurfaces of constant extrinsic curvature in any dimension. However in dimensions greater than 3 the smoothness condition on $\varphi$ plays an important role. In fact an example has been pointed out in [2] of an affine deformation of a uniform lattice in $\mathrm{SO}(3,1)$ which preserve no hypersurface in $\mathbb{R}^{3,1}$ with constant extrinsic curvature. By contrast in [18] it has been proved that any affine deformation of a uniform lattice in $\mathrm{SO}(3,1)$ preserves exactly one hypersurface of constant scalar curvature. 
Theorem A has been obtained as a consequence of more general statements about properly embedded spacelike surfaces in Minkowski 3-space of positive Gaussian curvature. Recall that there is a natural notion of Gauss map for spacelike surfaces in Minkowski space. In this context the Gauss map takes values on the hyperbolic plane, which is identified with the set of future-directed unit timelike vectors. We first prove that if the Gaussian curvature of $\Sigma$ is bounded by two positive constants then the image of the Gauss map is a domain of $\mathbb{H}^{2}$ bounded by geodesics. More specifically, by Corollary 1.17, the domain of dependence of $\Sigma$ is of the form $\mathcal{D}_{\varphi}$ for some lower semi-continuous function $\varphi: \partial \mathbb{H}^{2} \rightarrow \mathbb{R} \cup\{+\infty\}$.

Theorem $\mathrm{E}$ Let $\Sigma$ a properly embedded spacelike surface in $\mathbb{R}^{2,1}$ with Gaussian curvature bounded from above and below by positive constants. Let $\varphi: \partial \mathbb{H}^{2} \rightarrow \mathbb{R} \cup\{+\infty\}$ be such that the domain of dependence of $\Sigma$ is $\mathcal{D}_{\varphi}$. Then the Gauss map of $\Sigma$ is a diffeomorphism onto the interior of the convex hull of $\partial \mathbb{H}^{2} \backslash \varphi^{-1}(+\infty)$.

In Sect. 4 we will give a more precise version of this result, see Theorem 4.4. Notice in particular that the image of the Gauss map of a surface with Gaussian curvature bounded by two positive constants depends only on the domain of dependence of $\Sigma$. We will denote by $\Omega_{\varphi}$ the interior of the convex hull of $\partial \mathbb{H}^{2} \backslash \varphi^{-1}(+\infty)$. The second general result which we achieve, and which implies Theorem A, concerns the Minkowski problem. In general the Minkowski problem asks for a convex surface $\Sigma$ in $\mathbb{R}^{2,1}$ for which the domain $\Omega_{\Sigma}:=G_{\Sigma}(\Sigma)$ and the function $\psi:=\kappa_{\Sigma} \circ G_{\Sigma}^{-1}: \Omega_{\Sigma} \rightarrow \mathbb{R}_{>0}$ are prescribed. We will prove the following statement:

Theorem $\mathrm{F}$ Let $\mathcal{D}$ be a regular domain in $\mathbb{R}^{2,1}$ which is not a wedge, defined by a function $\varphi: \partial \mathbb{H}^{2} \rightarrow \mathbb{R} \cup\{+\infty\}$, and let $\psi$ be a continuous function defined on $\Omega_{\varphi}$ which is bounded by two positive constants. There exists a unique entire spacelike surface $\Sigma$ in $\mathcal{D}$ whose domain of dependence is $\mathcal{D}$ and whose curvature function satisfies:

$$
\kappa(p)=\psi \circ G_{\Sigma}(p)
$$

for every $p \in \Sigma$, where $G_{\Sigma}$ is the Gauss map of $\Sigma$.

Finally, we give an application of Theorem E to minimal Lagrangian maps between hyperbolic surfaces. The Gauss map of a CGC isometric immersion with $K=1$ into $\mathbb{R}^{2,1}$ is minimal Lagrangian: this means that it is area preserving and its graph is a minimal surface in the product. Conversely if $F: \Sigma \rightarrow \mathbb{H}^{2}$ is a minimal Lagrangian map with $\Sigma$ hyperbolic, one can produce an isometric immersion $\sigma_{F}: \Sigma \rightarrow \mathbb{R}^{2,1}$ such that $F$ coincides with the Gauss map of $\sigma_{F}$. Theorem $E$ states that if $\sigma_{F}$ is a proper embedding, then $F$ is injective and its image is a domain bounded by geodesics. As $\sigma_{F}$ is always a proper embedding if the domain is complete, we get the following corollary:

Corollary G Let $F: \mathbb{H}^{2} \rightarrow \mathbb{H}^{2}$ be a minimal Lagrangian map. Then $F$ is a diffeomeorphism onto the interior of the convex hull of $\overline{F\left(\mathbb{H}^{2}\right)} \cap \partial \mathbb{H}^{2}$. 


\section{Strategy of the proofs}

The support function $u_{\Sigma}$ (Definition 2.2) of a surface $\Sigma$ is a closed convex function (Definition 2.1) defined on the closed unit disk $\mathbb{D}$, the Klein model of $\overline{\mathbb{H}^{2}}$. If $\Sigma$ is properly embedded and has positive Gaussian curvature $\kappa(p)$ at every point $p$, then we show that the Gauss map $G_{\Sigma}$ is injective, $u_{\Sigma}$ is finite on the image of $G_{\Sigma}$. Moreover on this image $u_{\Sigma}$ satisfies the equation

$$
\operatorname{det} D^{2} u_{\Sigma}(\mathrm{x})=\frac{1}{\kappa\left(G_{\Sigma}^{-1}(\mathrm{x})\right)}\left(1-|\mathrm{x}|^{2}\right)^{-2}
$$

where $|x|$ is the Euclidean norm of $x$ in the disk [15]. The function $\varphi$ defining the domain of dependence of $\Sigma$ coincides with the restriction of $u_{\Sigma}$ to $\partial \mathbb{H}^{2}$.

The support function $u_{\Sigma}$ determines the surface $\Sigma$. In this way, Theorem F can be interpreted as the existence and uniqueness of solutions to a generalized Dirichlet problem for equation (1) with boundary data given by $\varphi$. It differs from the standard Dirichlet problem in that the boundary data $\varphi$ and the solution $u$ may both take the value $+\infty$. At the same time, the condition that $\Sigma$ be entire restricts the class of functions $u$ we consider to those that are gradient surjective (Definition 2.9).

This problem is made tractable by Theorem E and its more precise form Theorem 4.4. These theorems allow us to reduce our generalized Dirichlet problem on $\mathbb{D}$ to a problem on the smaller domain $\Omega_{\varphi}$, on which $u$ is necessarily finite.

The idea to prove Theorem E, or more generally Theorem 4.4, is to use a barrier argument: if $\Sigma$ is a surface with Gaussian curvature bounded by two positive constants, then for every boundary chord $c$ of $\Omega_{\varphi}$ we produce a convex surface $\Sigma^{\prime}$ so that

- $\Sigma^{\prime}$ lies above $\Sigma$;

- the image of the Gauss map of $\Sigma^{\prime}$ does not contain any point in the half plane $H$ bounded by $c$ in the complement of $\Omega_{\varphi}$.

The first point implies that the image of the Gauss map of $\Sigma$ is contained in the image of the Gauss map of $\Sigma^{\prime}$ so that the second point shows that $G_{\Sigma}(\Sigma)$ does not contain any point in $H$.

An important ingredient in the proof is the comparison principle for Monge-Ampère equations. However we have to apply the comparison principle to functions that are in general unbounded. So we need to prove a refined version of the comparison principle for convex functions that are possibly infinite at some points, which we do in Proposition 3.11. Here the hypothesis that $\Sigma$ is properly embedded plays a key role.

Once we have reduced Theorem $\mathrm{F}$ to a problem on $\Omega_{\varphi}$, we are able to produce a solution. But in order to prove that the corresponding CGC surface is entire, we need another barrier. Specifically, from the point of view of the surfaces themselves we need a lower barrier, or from the dual point of view of support functions we need an upper barrier. The general strategy follows the same line as in [4,9]. However, those papers use upper barriers invariant under a 1-parameter group, whereas such surfaces can never provide upper barriers for the general class of boundary values $\varphi$ that we consider. The support function of a barrier which is invariant under a one-parameter group must have boundary values which are finite on at least an open interval, whereas 
we consider functions $\varphi$ that are finite on as few as three points. Therefore we construct in Sect. 5 an entire CGC- $K$ for which $\varphi$ is finite at exactly three points, i.e. one whose domain of dependence is the intersection of the future of three null planes. This surface and the refined comparison principle are the key new ingredients to prove Theorem $\mathrm{F}$.

The construction of this particular surface is based on the harmonic maps $f_{ \pm}$: $\mathbb{C} \rightarrow \mathbb{H}^{2}$ with Hopf differential $\pm z d z^{2}$. It is known that the images of $f_{ \pm}$are open ideal triangles $T_{ \pm}$[13]. The map $F=f_{+} \circ f_{-}^{-1}: T_{-} \rightarrow \mathbb{H}^{2}$ is minimal Lagrangian and one studies the corresponding embedding $\sigma_{F}: T_{-} \rightarrow \mathbb{R}^{2,1}$. The embedding data of this surface are explicitly described in terms of the Hopf differential and the holomorphic energy of the harmonic map. The technical part is to show that the corresponding surface is properly embedded. Using the symmetry of the embedding $\sigma_{F}$ we reduce the problem to showing that the image of a line of symmetry is a properly embedded curve in Minkowski space. This is finally proved by studying the growth of the holomorphic energy of the harmonic map along the curve and its relation with the principal curvature of this isometric immersion. Once the barrier (which we will call a triangular surface) is fully described, the Minkowski problem is considered.

For a given lower semicontinuous function $\varphi: \partial \mathbb{H}^{2} \rightarrow \mathbb{R} \cup\{+\infty\}$ and a bounded continuous function $\psi$ defined on the interior $\Omega_{\varphi}$ of the convex hull of $\varphi^{-1}(\mathbb{R})$ we construct a function $u$ on $\overline{\Omega_{\varphi}}$ which solves the equation

$$
\operatorname{det} D^{2} u(\mathrm{x})=\frac{1}{\psi(\mathrm{x})}\left(1-|\mathrm{x}|^{2}\right)^{-2}
$$

and is the linear interpolation of $\varphi$ on the boundary of $\Omega_{\varphi}$. To the end we consider the convex envelope $\operatorname{conv}(\varphi)$ of $\varphi$. Taking an interior approximation $\Omega_{n}$ of $\Omega_{\varphi}$ by convex domains, we consider the solution $u_{n}$ of the Eq. (2) on $\Omega_{n}$ with boundary data $\operatorname{conv}(\varphi)$. Applying the comparison principle with classical barriers we prove that $u_{n}$ converges to the solution of the problem. The function $u$ defines a spacelike convex surface $\Sigma$ in Minkowski space, that must be proved to be entire. More precisely $\Sigma$ is part of an entire achronal surface, which however could contain some additional regions which are not strictly convex. The problem reduces to showing that $\bar{\Sigma}$ does not meet any plane $P$ whose normal vector lies in $\partial \Omega_{\varphi}$. In fact for such a $P$ we will show that there is a triangular surface which separates $\Sigma$ from $P$. The proof of this fact is based again on the comparison principle.

\section{Organization of the paper}

Sections 1, 2, and 3 contain preliminaries as well as proofs of some general theorems for which we could not find references. In Sect. 1 we quickly review the theory of spacelike surfaces in Minkowski space. First and second fundamental forms are introduced and the relevant Gauss-Codazzi equations explained. We show that properly embedded spacelike surfaces are graph, and introduce the notion of domain of dependence. We will see that aside from few exceptions the domain of dependence is a regular domain. In Sect. 2, the notion of support function is given and the relation between the boundary value of the support function and the domain of dependence of the surface is pointed out. The relation between curvature of the surface and the 
support function is described, and Minkowski problem is shown to be equivalent to a Dirichlet problem for a Monge-Ampère equation. In Sect. 3 we describe the analytical tools we will need to solve our problem. Classical results of stability for solutions of Monge-Ampère equations are given and a refined version of the comparison principle for unbounded functions is proved.

The remainder of the paper contains our main results on CGC surfaces. In Sect. 4 we prove Theorem E. First we study some special CGC surfaces whose domain of dependence is the future of a spacelike half-line in Minkowski space. Those surfaces and our comparison principle will be the key ingredients to prove Theorem E. In Sect. 5 we study the triangular surfaces. First we construct the embedding data of a CGC immersion on $\mathbb{C}$ by means of a correspondence with harmonic maps and minimal Lagrangian maps. Then we prove that this immersion is a proper embedding. Section 6 is devoted to solving the Minkowski problem. As an application we will prove in Sect. 7 that any regular domain $\mathcal{D}$ which is not a wedge is foliated by CGC surfaces.

Finally in Sect. 8 we point out an open question.

\section{Spacelike surfaces in Minkowski space}

Minkowski $(2+1)$-space is the simply connected geodesically complete flat Lorentzian manifold $\mathbb{R}^{2,1}=\left(\mathbb{R}^{3}, d x_{1}^{2}+d x_{2}^{2}-d x_{3}^{2}\right)$. A nonzero tangent vector $\boldsymbol{v}$ is called spacelike, lightlike or timelike if $\langle\boldsymbol{v}, \boldsymbol{v}\rangle>0,\langle\boldsymbol{v}, \boldsymbol{v}\rangle=0$ or $\langle\boldsymbol{v}, \boldsymbol{v}\rangle<0$ respectively. We also say $\boldsymbol{v}$ is causal if it is either lightlike or timelike, and $\boldsymbol{v}$ is achronal if it is either lightlike or spacelike. A causal vector is either future-directed if its $x_{3}$-component is positive and past-directed if its $x_{3}$-component is negative.

A point $\boldsymbol{p}$ is in the future of $\boldsymbol{q}$ (and $\boldsymbol{q}$ is in the past of $\boldsymbol{p}$ ) if $\boldsymbol{p}-\boldsymbol{q}$ is timelike future-directed. We denote by $I^{+}(\boldsymbol{p})$ (resp. $I^{-}(\boldsymbol{p})$ ) the open cone of points in the future (resp. past) of $\boldsymbol{p}$. If $S$ is any set in Minkowski space, we then define the future and past of $S$ as

$$
I^{+}(S)=\bigcup_{\boldsymbol{p} \in S} I^{+}(\boldsymbol{p}) \quad \text { and } \quad I^{-}(S)=\bigcup_{\boldsymbol{p} \in S} I^{-}(\boldsymbol{p})
$$

and we say $S$ is future-complete if $I^{+}(S) \subset S$.

A $C^{0}$ submanifold $\Sigma$ is causal (resp. achronal) if for each point $\boldsymbol{p} \in \Sigma$, there is a neighborhood of $\boldsymbol{p}$ in which every point of $\Sigma$ is causally (resp. achronally) separated from $\boldsymbol{p}$. For some of the preliminaries we allow immersed surfaces, in which case "locally" means locally in the domain; however for the bulk of the paper we are concerned only with entire surfaces, which are necessarily embedded. A $C^{1}$ surface is spacelike, lightlike, or timelike if the induced metric on the tangent space is positive definite, degenerate, or indefinite respectively. If $\Sigma \subset \mathbb{R}^{2,1}$ is a $C^{1}$ spacelike surface, the future unit normal vector field is the unique future-directed vector field $\boldsymbol{n}$ orthogonal to $\Sigma$ such that $\langle\boldsymbol{n}, \boldsymbol{n}\rangle=-1$.

The purpose of the following section is to introduce preliminary geometric notions on spacelike surfaces in $\mathbb{R}^{2,1}$, including the definition of entire surface and of domain of dependence, and finally state the Minkowski and CGC problems which are the main focus of this paper. 


\subsection{Embedding data for spacelike surfaces}

Let us denote by $D$ the flat connection of $\mathbb{R}^{3}$. For a smoothly immersed spacelike surface $\Sigma$ in $\mathbb{R}^{2,1}$ we recall:

- The first fundamental form $\mathrm{I}$ is the Riemannian metric on $T \Sigma$ given by the restriction of the metric $\langle\cdot, \cdot\rangle$.

- The Levi-Civita connection $\nabla$ and second fundamental form II are defined on $T \Sigma$ as the tangential and normal components respectively of the connection $D$ :

$$
D_{v} \boldsymbol{w}=\nabla_{\boldsymbol{v}} \boldsymbol{w}+\mathrm{II}(\boldsymbol{v}, \boldsymbol{w}) \boldsymbol{n} .
$$

- The shape operator $B$ is the self-adjoint endomorphism of $T \Sigma$ given by differentiating the normal vector field $n$ :

$$
B(\boldsymbol{v})=D_{\boldsymbol{v}}(\boldsymbol{n}) .
$$

The three objects I, II, and $B$ are related by the Weingarten equation $\operatorname{II}(\boldsymbol{v}, \boldsymbol{w})=$ $\mathrm{I}(B(\boldsymbol{v}), \boldsymbol{w})$. The third fundamental form III is defined by $\operatorname{III}(\boldsymbol{v}, \boldsymbol{w})=\mathrm{I}(B(\boldsymbol{v}), B(\boldsymbol{w}))$. Moreover, the pair (I, B) satisfies the Gauss equation:

$$
\kappa_{I}=-\operatorname{det} B
$$

where $\kappa_{I}$ is the intrinsic curvature of I, and the Codazzi equation:

$$
d^{\nabla} B=0
$$

where $d^{\nabla}$ is the extension of $\nabla$ to $T \Sigma$-valued differential forms, which is given by the formula (equivalent to the vanishing of the torsion of $\nabla$ ):

$$
d^{\nabla} B(\boldsymbol{v}, \boldsymbol{w})=\left(\nabla_{\boldsymbol{v}} B\right)(\boldsymbol{w})-\left(\nabla_{\boldsymbol{w}} B\right)(\boldsymbol{v}) .
$$

The Fundamental Theorem of surface theory, in the case of Minkowski space, shows that Eqs. (3) and (4) also provide sufficient conditions to determine, at least for a simply connected surface $\Sigma$, a spacelike immersion into $\mathbb{R}^{2,1}$ :

Theorem 1.1 Let $\Sigma$ be a simply connected surface. Given a Riemannian metric I on $\Sigma$ and $a(1,1)$-tensor $B \in \Gamma(\operatorname{End}(T \Sigma))$, self-adjoint for $\mathrm{I}$, such that the pair $(\mathrm{I}, B)$ satisfies Eqs. (3) and (4), there exists a spacelike immersion $\sigma: \Sigma \rightarrow \mathbb{R}^{2,1}$ such that the pull-back of the first fundamental form and shape operator of $\sigma(\Sigma)$ coincide with $\mathrm{I}$ and B. Moreover, any two such immersions differ by post-composition with a global isometry of $\mathbb{R}^{2,1}$.

We define the Gaussian curvature in an extrinsic way:

Definition 1.2 The Gaussian curvature of $\Sigma$ is det B. A surface with constant Gaussian curvature equal to $K$ is called $C G C-K$. 


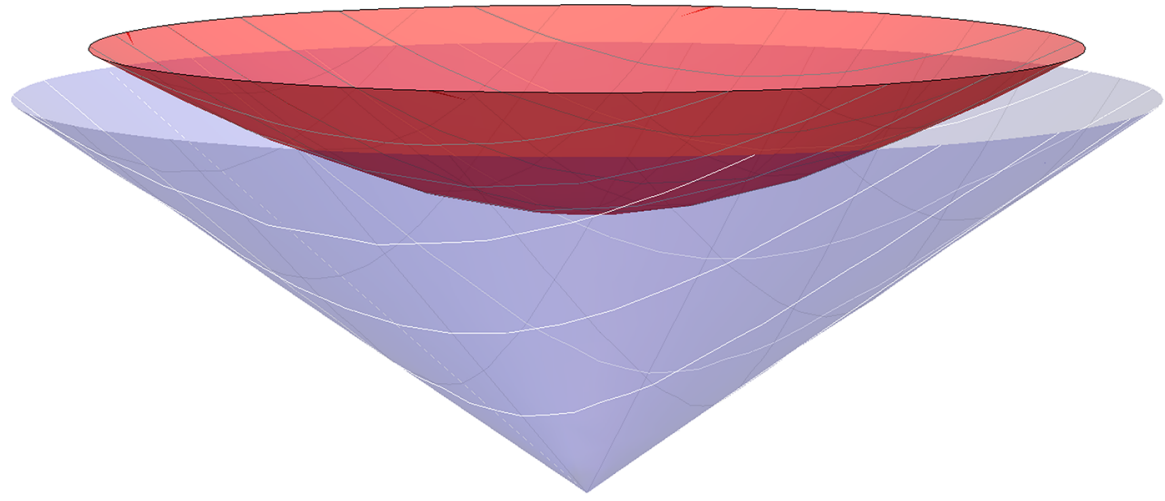

Fig. 1 The hyperboloid Hyp, whose domain of dependence is the cone $I^{+}(\mathbf{0})$

By Gauss' equation (3), $\Sigma$ is a CGC- $K$ surface if and only if the first fundamental form has constant intrinsic curvature $-K$.

Example 1.3 (See Fig. 1) The future sheet of the two-sheeted hyperboloid

$$
\text { Hyp }:=\left\{x \in \mathbb{R}^{2,1}:\langle x, x\rangle=-1, x_{3}>0\right\},
$$

is CGC-1. Since it is simply connected and the first fundamental form I is a complete hyperbolic metric (i.e. of constant intrinsic curvature -1), Hyp is isometric to the hyperbolic plane $\mathbb{H}^{2}$. The normal vector of Hyp at a point $\boldsymbol{p}$ is $\boldsymbol{n}(\boldsymbol{p})=\boldsymbol{p}$, hence the shape operator of Hyp is the identity. When considered as a surface in its own right we will use the notation Hyp and when viewed as the target of the Gauss map (see below) of any surface, we will refer to it as $\mathbb{H}^{2}$.

Example 1.4 Define the trough $T$ by

$$
T:=\left\{x \in \mathbb{R}^{2,1}: x_{2}^{2}-x_{3}^{2}=-1, x_{3}>0\right\} .
$$

It can be described as the cartesian product of a hyperbola $x_{2}^{2}-x_{3}^{2}=-1$ and a line. The eigenvalues of the shape operator of $T$ are 1 and 0 , so it has zero Gaussian curvature. See Fig. 2.

The Gauss map of a $C^{1}$ spacelike surface $\Sigma$, analogously to the Euclidean case, is the function

$$
G_{\Sigma}: \Sigma \rightarrow \mathbb{H}^{2}
$$

defined by

$$
G_{\Sigma}(p)=n(p),
$$




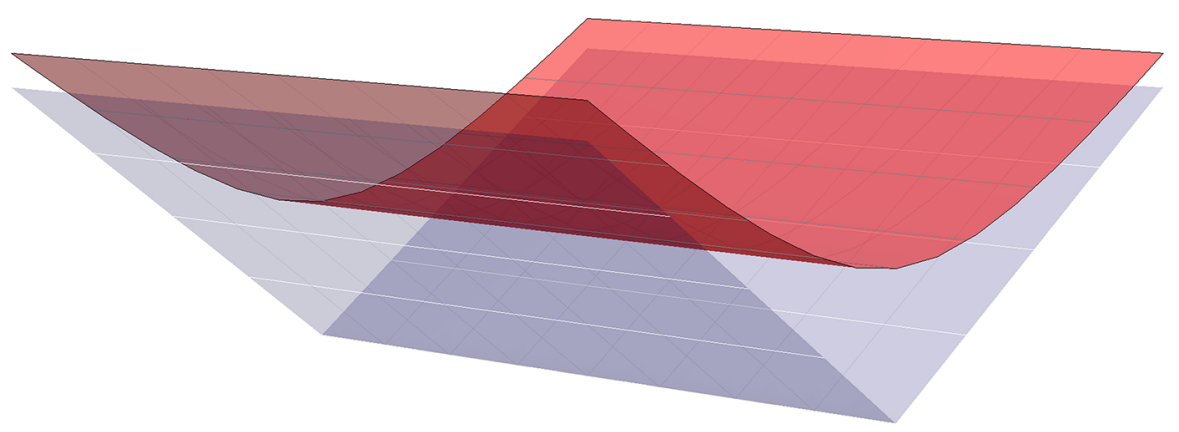

Fig. 2 The trough $T$, whose domain of dependence is the wedge $I^{+}(\ell)$

where $\boldsymbol{n}$ is the future unit normal vector of $\Sigma$, considered as a point of $\mathbb{H}^{2}$. Since the shape operator $B$ is the derivative of the Gauss map, the third fundamental form is the pull back under $G_{\Sigma}$ of the hyperbolic metric $h_{\mathbb{H}^{2}}$ on $\mathbb{H}^{2}$.

\subsection{CGC surfaces and minimal Lagrangian maps}

Let us now explain the relation between surfaces of constant Gaussian curvature and minimal Lagrangian diffeomorphisms between hyperbolic surfaces.

Definition 1.5 Given two hyperbolic surfaces $(S, h)$ and $\left(S^{\prime}, h^{\prime}\right)$, a diffeomorphism $F$ : $(S, h) \rightarrow\left(S^{\prime}, h^{\prime}\right)$ is minimal Lagrangian if the unique positive definite $h$-symmetric tensor $b \in \Gamma(\operatorname{End}(T S))$ such that $F^{*} h^{\prime}=h(b \cdot, b \cdot)$ satisfies the Codazzi equation $d^{\nabla_{h}} b=0$, where $\nabla_{h}$ is the Levi-Civita connection of $h$.

Remark 1.6 The tensor $b$ can also be described as the symmetric part of the polar decomposition of the linear map $d F$ with respect to the inner products $h$ and $h^{\prime}$. If $F$ is a minimal Lagrangian map between hyperbolic surfaces, it follows that det $b=1$ [14].

Lemma 1.7 Given any convex $C G C-K$ surface $\Sigma^{K}$ in $\mathbb{R}^{2,1}$, with first fundamental form I, the Gauss map of $\Sigma^{K}$ is a minimal Lagrangian map, when considered as a map:

$$
G:\left(\Sigma^{K}, K \cdot \mathrm{I}\right) \rightarrow\left(\mathbb{H}^{2}, h_{\mathbb{H}^{2}}\right)
$$

Proof First of all, observe that, by Gauss' equation (3) the intrinsic curvature of I equals $-K$, and therefore the metric $K \cdot \mathrm{I}$ is a hyperbolic metric. Now, let us take $b=(1 / \sqrt{K}) B$. The pull-back of the hyperbolic metric of $\mathbb{H}^{2}$ by the Gauss map is:

$$
G^{*} h_{\mathbb{H}^{2}}(v, w)=\operatorname{III}(v, w)=\mathrm{I}(B(v), B(w))=K \mathrm{I}(b(v), b(w)),
$$

where $B=D n$ is the shape operator of $\Sigma$. Moreover, since $B$ is self-adjoint and Codazzi for I, then it is also self-adjoint and Codazzi for $K \cdot \mathrm{I}$, and so is $b$. 
Lemma 1.8 Given a simply connected hyperbolic surface $(S, h)$, possibly not complete, and a minimal Lagrangian local diffeomorphism $F:(S, h) \rightarrow \mathbb{H}^{2}$, there exists an isometric immersion $\sigma:(S,(1 / K) \cdot h) \rightarrow \mathbb{R}^{2,1}$ with Gauss map equal to $F$.

Proof Let $b$ be as in Definition 1.5. Then, the proof of Lemma 1.7 suggests the ansatz $((1 / K) \cdot h, \sqrt{K} b)$ for the embedding data of a CGC- $K$ surface. It then follows from Remark 1.6 that the pair $((1 / K) \cdot h, \sqrt{K} b)$ satisfies the equations of Gauss and Codazzi. Hence by Theorem 1.1 , there exists an immersion $\sigma$ having $((1 / K) \cdot h, \sqrt{K} b)$ as embedding data.

Moreover, from the definition of $b$ we have $F^{*} h_{\mathbb{H}^{2}}=h(b \cdot, b \cdot)$, while from the same computation as in the proof of Lemma $1.7, G_{\sigma}^{*} h_{\mathbb{H}^{2}}=(1 / K) \cdot h(\sqrt{K} b \cdot \sqrt{K} b \cdot)=$ $h(b \cdot, b \cdot)$. Hence at each point $\boldsymbol{p} \in S, F$ and $G$ differ by an isometry of $\mathbb{H}^{2}$ in a neighborhood of $\boldsymbol{p}$. Since $S$ is connected, this isometry must in fact be constant. By postcomposing $\sigma$ with the corresponding isometry of $\mathbb{R}^{2,1}$, we may take it to be the identity.

\subsection{Entire spacelike surfaces}

In this paper, we will study entire embedded spacelike surfaces. Let us introduce this notion.

Definition 1.9 An achronal surface in $\mathbb{R}^{2,1}$ is entire if $\left.\pi\right|_{\Sigma}: \Sigma \rightarrow \mathbb{R}^{2}$ is a homeomorphism, where $\pi: \mathbb{R}^{2,1} \rightarrow \mathbb{R}^{2}$ is the vertical projection $\pi\left(x_{1}, x_{2}, x_{3}\right)=\left(x_{1}, x_{2}\right)$.

Entire achronal surfaces are exactly the graphs of 1-Lipschitz functions on $\mathbb{R}^{2}$. Entire spacelike surfaces are exactly the graphs of $C^{1}$ and strictly 1-Lipschitz functions on $\mathbb{R}^{2}$. Clearly an entire surface is properly immersed. The following elementary proposition says that the converse is true as well.

Proposition 1.10 Every properly immersed achronal surface in $\mathbb{R}^{2,1}$ is entire.

Proof Let $\Sigma$ be a properly immersed achronal surface. By the achronal condition, the projection $\pi: \Sigma \rightarrow \mathbb{R}^{2}$ is a local homeomorphism. We now prove that $\pi$ has the path lifting property: given a point $\boldsymbol{p}$ is $\Sigma$ and a curve $\gamma:[0,1] \rightarrow \mathbb{R}^{2}$ with $\gamma(0)=\pi(\boldsymbol{p})$, there is a lift $\tilde{\gamma}:[0,1] \rightarrow \Sigma$ with $\pi \circ \tilde{\gamma}=\gamma$. Let $\gamma:[0,1] \rightarrow \mathbb{R}^{2}$ be such a curve. Since $\pi$ is a local homeomorphism, the path $\gamma$ can be lifted to an open neighborhood. Since $\Sigma$ is achronal, the length of any partial lift $\tilde{\gamma}$ measured using the Euclidean metric on $\mathbb{R}^{3}$ is at most $\sqrt{2}$ times the length of $\gamma$ in $\mathbb{R}^{2}$. Since the immersion is proper, the induced Euclidean metric on $\Sigma$ is complete. As a consequence, the partial lift of $\gamma$ can also be extended to all limit points. Therefore the interval on which we can lift $\gamma$ is both open and closed, so it is the entire interval $[0,1]$.

We have shown that $\pi$ is a local homeomorphism with the path lifting property, so it is a covering map [7, p. 383]. But the image $\mathbb{R}^{2}$ is simply connected, so $\pi$ must be a homeomorphism.

Remark 1.11 Proposition 1.10 shows that the condition of being entire is preserved by isometries of $\mathbb{R}^{2,1}$. In other words, if $\left.\pi\right|_{\Sigma}$ is a homeomorphism, then the orthogonal projection from $\Sigma$ to any spacelike plane is a homeomorphism. 
Remark 1.12 The projection $\pi$ is distance non-decreasing. Therefore, if the first fundamental form of a spacelike surface $\Sigma$ is a complete Riemannian metric, then $\Sigma$ is necessarily entire. The converse is false; a counterexample will be provided by the entire surface studied in Sect. 5, whose fundamental form is isometric to an ideal triangle in $\mathbb{H}^{2}$. See also [4, Appendix A] for another counterexample.

\subsection{Domains of dependence}

Recall that a continuous curve $\gamma: I \rightarrow \mathbb{R}^{2,1}$ is called causal if for all pairs of points $t, s \in I$, the images $\gamma(t)$ and $\gamma(s)$ differ by a lightlike or timelike vector.

Definition 1.13 Given a spacelike surface $\Sigma$ in $\mathbb{R}^{2,1}$, the domain of dependence $\mathcal{D}_{\Sigma}$ of $\Sigma$, is the set of all points $\boldsymbol{p} \in \mathbb{R}^{2,1}$ such that every inextendable causal curve through $p$ intersects $\Sigma$.

Let us provide the following description of domains of dependence for entire spacelike surfaces. We say that a half-space is null if it is bounded by a lightlike plane. An open null half-space is equal either to the future or to the past of its boundary plane.

Lemma 1.14 If $\Sigma$ is an entire spacelike surface in $\mathbb{R}^{2,1}$, then its domain of dependence $\mathcal{D}_{\Sigma}$ is open, and is equal to the intersection of the open null half-spaces containing $\Sigma$. Moreover, exactly one of the following holds:

(1) $\mathcal{D}_{\Sigma}=\mathbb{R}^{2,1}$;

(2) $\mathcal{D}_{\Sigma}=I^{+}(Q) \cap I^{-}(P)$ where $Q$ and $P$ are parallel null planes, with $P$ lying in the future of $Q$;

(3) $\mathcal{D}_{\Sigma}=\bigcap_{Q \in \mathcal{F}} I^{+}(Q)$ where $\mathcal{F}$ is a nonempty family of null planes; or

(4) $\mathcal{D}_{\Sigma}=\bigcap_{Q \in \mathcal{F}} I^{-}(Q)$ where $\mathcal{F}$ is a nonempty family of null planes.

Proof We divide the proof into several steps.

Step 1: We first prove that $\mathcal{D}_{\Sigma}$ is open. Let Caus $\mathbb{R}_{\mathbb{R}^{2,1}}$ be the space of all inextendable causal curves in $\mathbb{R}^{2,1}$, with the topology of local uniform convergence. Since every such curve is the graph of a 1-Lipschitz function from $\mathbb{R}$ to $\mathbb{R}^{2}$, the Arzelà-Ascoli theorem implies that for any compact set $K \in \mathbb{R}^{2,1}$, the subset Caus ${ }_{K}$ of such curves intersecting $K$ is compact. We now show that the set Caus ${ }_{\Sigma}$ of such curves intersecting $\Sigma$ is open. Suppose $\gamma \in \operatorname{Caus}_{\mathbb{R}^{2,1}}$ intersects $\Sigma$ at a point $\boldsymbol{p}$. Since $\Sigma$ is spacelike, a small circle in $\Sigma$ around $\boldsymbol{p}$ must be at least some fixed Euclidean distance from the light cone of $\boldsymbol{p}$. Perturbing $\gamma$ by less than this distance, it must still pass through the circle and hence intersect $\Sigma$.

To complete the proof that $\mathcal{D}_{\Sigma}$ is open, for any $p \in \mathcal{D}_{\Sigma}$, let $K_{n}$ be a sequence of compact neighborhoods of $\boldsymbol{p}$ in $\mathbb{R}^{2,1}$ whose intersection is $\boldsymbol{p}$. Then Caus $\boldsymbol{p}=$ $\bigcap_{n} \operatorname{Caus}_{K_{n}} \subset$ Caus $_{\Sigma}$. Since Caus $K_{n}$ are compact and Caus $\Sigma$ is open it follows that for $n$ sufficiently large, Caus $_{K_{n}} \subset$ Caus $_{\Sigma}$ whence $K_{n} \subset \mathcal{D}_{\Sigma}$.

Step 2: We show that every open null half-space containing $\Sigma$ also contains $\mathcal{D}_{\Sigma}$. Let $H$ be an open null half-space containing $\Sigma$. For any point $\boldsymbol{p} \notin H$, the null line through $p$ parallel to the boundary of $H$ lies entirely outside of $H$. Since $\Sigma$ is contained in $H$, this line exhibits a causal curve containing $\boldsymbol{p}$ which does not meet $\Sigma$, showing that $\boldsymbol{p} \notin \mathcal{D}_{\Sigma}$. Therefore $\mathcal{D}_{\Sigma} \subset H$. 
Step 3: We prove that $\mathcal{D}_{\Sigma}$ is the intersection of the open null half-spaces containing $\Sigma$. By Step $2, \mathcal{D}_{\Sigma}$ is contained in this intersection. Now we simply need to show that if $\boldsymbol{p} \notin \mathcal{D}_{\Sigma}$, we can find a closed null half space containing $\boldsymbol{p}$ but not $\Sigma$.

Let $\boldsymbol{p}$ be a point not in $\mathcal{D}_{\Sigma}$. Since $\Sigma \subset \mathcal{D}_{\Sigma}, \boldsymbol{p}$ is either in the past or the future of $\Sigma$. If $\boldsymbol{p}$ is in the past of $\Sigma$, then any point in $\overline{I^{-}(\boldsymbol{p})}$ can be connected to $p$ by a causal geodesic which does not meet $\Sigma$. Hence if one can "escape" $\Sigma$ from $p$, one can also escape $\Sigma$ from any point in the past of $\boldsymbol{p}$, so all of $\overline{I^{-}(\boldsymbol{p})}$ must be outside of $\mathcal{D}_{\Sigma}$. Similarly, if $\boldsymbol{p} \in I^{+}(\Sigma)$, then all of $\overline{I^{+}(\boldsymbol{p})}$ must lie outside of $\mathcal{D}_{\Sigma}$.

Up to time reversal, we may assume that $p \in I^{-}(\Sigma)$. Let $\boldsymbol{q}$ be a point in $\overline{I^{+}(\boldsymbol{p})}$ which is still below $\Sigma$ but is contained in the boundary of $\mathcal{D}_{\Sigma}$ (it may be that $\boldsymbol{q}=\boldsymbol{p}$ ). Since $\boldsymbol{q} \notin \mathcal{D}_{\Sigma}$, there is an inextendable causal curve $\gamma$ containing $\boldsymbol{q}$ which does not intersect $\Sigma$. We first show that the part $\gamma^{+}$of $\gamma$ in the closed future of $\boldsymbol{q}$ must be a null geodesic ray. Otherwise, it would contain a point $\boldsymbol{r}$ which was timelike separated from $\boldsymbol{q}$, and so by the previous paragraph, $\overline{I^{-}(\boldsymbol{r})}$ would be disjoint from $\mathcal{D}_{\Sigma}$. But $I^{-}(\boldsymbol{r})$ contains an open neighborhood of $\boldsymbol{q}$, which contradicts $\boldsymbol{q} \in \partial \mathcal{D}_{\Sigma}$.

Let $H=I^{-}\left(\gamma^{+}\right)$. This is the unique open past-complete null half-space containing $\gamma^{+}$in its boundary. By the same reasoning as above, $H$ cannot intersect $\mathcal{D}_{\Sigma}$, and since $\mathcal{D}_{\Sigma}$ is open, neither can $\bar{H}$. But $\boldsymbol{q}$ and $\boldsymbol{p}$ are both in $\bar{H}$, which completes the proof.

Step 4: We prove that exactly one of the four options must hold. It is enough to observe that if $\mathcal{D}_{\Sigma}$ is contained in the intersection of a past-complete null half-space $\mathrm{H}^{-}$ and a future-complete null half-space $H^{+}$then the boundaries of $H^{+}$and $H^{-}$must be parallel. Otherwise, the projection of $\mathcal{D}_{\Sigma}$ to $\mathbb{R}^{2}$ could not be surjective, but it must be since $\Sigma \subset \mathcal{D}_{\Sigma}$ and $\Sigma$ is entire.

We have the following definition of future-complete domains and future-convex spacelike surfaces.

Definition 1.15 An entire achronal surface $\Sigma$ is called future-convex (resp. strictly future-convex) if $I^{+}(\Sigma)$ is future-complete and convex (resp. strictly convex).

Remark 1.16 The condition that a $C^{2}$ entire spacelike surface $\Sigma$ is future-convex is equivalent to the fact that the shape operator $B=D \boldsymbol{n}$ (where $\boldsymbol{n}$ is the future unit normal vector field) is positive semi-definite. Hence these are surfaces having non-negative mean curvature and Gaussian curvature, namely $\operatorname{tr} B \geq 0$ and $\operatorname{det} B \geq 0$.

From Lemma 1.14, we therefore have the following characterization of domains of dependence of future-convex entire surfaces:

Corollary 1.17 If $\Sigma$ is a future-convex entire spacelike surface in $\mathbb{R}^{2,1}$, then $\mathcal{D}_{\Sigma}$ is a convex open domain of the form

$$
\mathcal{D}_{\Sigma}=\bigcap_{Q \in \mathcal{F}} I^{+}(Q),
$$

where $\mathcal{F}$ is a (possibly empty) family of null planes. We can take $\mathcal{F}$ to be the family of all null planes containing $\Sigma$ in their future. 
Remark 1.18 There is clearly an analogous definition of past-complete domains and past-convex surfaces. Any isometry of $\mathbb{R}^{2,1}$ which is not future-preserving exchanges future-complete domains with past-complete domains, and future-convex surfaces with past-convex surfaces. For this reason, we will always assume without loss of generality that our surfaces are future-convex with future-complete domains of dependence.

Example 1.19 The domain of dependence of the hyperboloid Hyp of Example 1.3 is the future cone over the origin, namely:

$$
\mathcal{D}_{\mathbb{H}^{2}}=I^{+}(\mathbf{0})
$$

This is the intersection of all the future half-spaces bounded by a null plane through the origin.

Example 1.20 The domain of dependence of the trough $T$ of Example 1.4 is the wedge:

$$
W=I^{+}\left(Q^{1}\right) \cap I^{+}\left(Q^{2}\right),
$$

where $Q^{1}$ and $Q^{2}$ are two non-parallel planes, which intersect along a spacelike line $\ell$. Namely, $\partial W$ is composed of two null half-planes, both having the same spacelike line $\ell$ as a boundary. See again Fig. 2 .

Let us observe that, if the family $\mathcal{F}$ of Corollary 1.17 is empty, then $\mathcal{D}_{\Sigma}=\mathbb{R}^{2,1}$, while if $\mathcal{F}$ contains only one element $Q$, then $\mathcal{D}_{\Sigma}$ is the future of the null plane $Q$. In Example 1.20, we can assume $\mathcal{F}$ is composed of exactly two non-parallel null planes. We will say that $\mathcal{D}_{\Sigma}$ is a (future-complete) regular domain if $\mathcal{F}$ contains at least two non-parallel elements. More precisely:

Definition 1.21 A convex open domain $\mathcal{D} \subset \mathbb{R}^{2,1}$ is a regular domain if

$$
\mathcal{D}=\bigcap_{Q \in \mathcal{F}} I^{+}(Q),
$$

for some family $\mathcal{F}$ of null planes which contains at least two non-parallel distinct planes.

\subsection{Minkowski problem in regular domains}

With these preliminary remarks in hand, we can formulate more precisely the statement of the problems we consider in this paper. Let us denote by $\kappa_{\Sigma}: \Sigma \rightarrow \mathbb{R}$ the Gaussian curvature of a spacelike surface $\Sigma$. The Minkowski problem we consider can be stated as follows:

Minkowski problem Given any regular domain $\mathcal{D}$ in $\mathbb{R}^{2,1}$ and any sufficiently regular function $\psi: \mathbb{H}^{2} \rightarrow \mathbb{R}^{>0}$, does there exist a unique entire surface $\Sigma$ such that

(1) $\psi \circ G_{\Sigma}=\kappa_{\Sigma}$, and 
(2) $\mathcal{D}_{\Sigma}=\mathcal{D}$ ?

We will give a positive answer (Theorem F) to the Minkowski problem, under the assumption that $\mathcal{D}$ is not a wedge (compare Example 1.20), which we will show is also a necessary condition.

Remark 1.22 Let us make some remarks on the formulation of the problem.

(1) Consistently with the classical Minkowski problem in Euclidean space, we will consider the Minkowski problem for a prescribed positive function $\psi$ on $\mathbb{H}^{2}$. This implies that a surface $\Sigma$ is strictly convex-that is, either $I^{+}(\Sigma)$ or $I^{-}(\Sigma)$ is a strictly convex domain (with smooth boundary equal to $\Sigma$ ).

(2) We will give an affirmative answer to the Minkowski problem-both for the existence and uniqueness part-under the assumption that

$$
a<\psi<b
$$

for some constants $a, b>0$. Without such assumption, the problem appears significantly more complicated, at least with the tools of this paper and of the existing literature.

(3) We shall prove in Sect. 4 that, for every entire spacelike surface $\Sigma$ with Gaussian curvature bounded from above and below by positive constants (as in the previous point), the image of the Gauss map $G_{\Sigma}$ coincides with the image of the subdifferential of $\partial \mathcal{D}_{\Sigma}$ - that is, with the set of vectors $v \in \mathbb{H}^{2}$ such that $\mathcal{D}_{\Sigma}$ admits a support plane orthogonal to $\boldsymbol{v}$. Hence the function $\psi$ need only be defined on the image of the Gauss map of $\partial \mathcal{D}_{\Sigma}$.

(4) If $\Sigma$ is a strictly convex smooth entire surface in $\mathbb{R}^{2,1}$, then its Gauss map $G_{\Sigma}$ : $\Sigma \rightarrow \mathbb{H}^{2}$ is a diffemorphism onto its image. Hence under our assumptions, the condition of the Minkowski problem can also written as

$$
\psi=\kappa_{\Sigma} \circ G_{\Sigma}^{-1}
$$

The function $\kappa_{\Sigma} \circ G_{\Sigma}^{-1}$ is also called curvature function of $\Sigma$.

A particular case is obtained when the prescribed curvature function is constant. In Theorem A we will give a positive answer, under the necessary and sufficient condition that the regular domain $\mathcal{D}$ is not a wedge, to the following problem:

CGC problem Given any regular domain $\mathcal{D}$, does there exist for every $K>0$ a unique entire CGC-K surface $\Sigma^{K}$ such that its domain of dependence $\mathcal{D}_{\Sigma^{K}}$ is the prescribed regular domain $\mathcal{D}$ ?

\section{Analytical formulation}

The purpose of this section is to translate the study of convex surfaces in Minkowski space in analytical terms, with particular focus on the aforementioned Minkowski problem. That is, we introduce the support function for convex spacelike surfaces 
and we express the Minkowski problem in terms of a partial differential equation of Monge-Ampère type.

\subsection{Support functions}

It will be convenient to introduce the following definitions from the theory of convex functions:

Definition 2.1 [16] A function $\mathbb{R}^{n} \rightarrow \mathbb{R} \cup\{ \pm \infty\}$ is called convex, resp. closed, if its supergraph $\left\{(\mathrm{x}, z) \subset \mathbb{R}^{n} \times \mathbb{R} \mid f(\mathrm{x})<+\infty\right.$ and $\left.z \geq f(\mathrm{x})\right\}$ is convex, resp. closed. A function $f$ is proper if $f(\mathbf{x})<+\infty$ for at least one $\mathbf{x}$ and $f(\mathbf{x})>-\infty$ for every $\mathbf{x}$. The essential support of a convex function $f$ is the set on which $f$ is finite.

Except for minor technicalities, we are concerned only with proper functions. However, it is essential that we consider functions which take the value $+\infty$ at some points, so we will henceforth allow all our functions to be infinite without further ado. Note that a function is closed if and only if it is lower semi-continuous. If $X$ is a subset of $\mathbb{R}^{n}$, we will say $f$ is a function on $X$ if it is a (proper) function on $\mathbb{R}^{n}$ with essential support contained in $X$.

In the following definition, we are interested especially in the case where the set $S$ is a future-convex entire spacelike surface and the case where $S$ is a domain of dependence.

Definition 2.2 Let $S$ be a nonempty subset of $\mathbb{R}^{2,1}$. Then the support function of $\mathrm{S}$ is the function $u_{S}: \overline{\mathbb{D}} \rightarrow \mathbb{R} \cup\{+\infty\}$ defined by

$$
u_{S}(\mathrm{y})=\sup _{\boldsymbol{x} \in S}\langle\boldsymbol{x},(\mathrm{y}, 1)\rangle
$$

where $\overline{\mathbb{D}}$ is the closed unit disk in $\mathbb{R}^{2}$ and $\mathrm{y}=\left(y_{1}, y_{2}\right) \in \overline{\mathbb{D}}$.

Observe that the plane $\left\{x \in \mathbb{R}^{2,1} \mid\langle\boldsymbol{x},(\mathrm{y}, 1)\rangle=z\right\}$ is spacelike for $\mathrm{y}$ in the interior of the unit disk and null for $y$ on the unit circle. In fact, as $y$ and $z$ range over $\overline{\mathbb{D}} \times \mathbb{R}$, this parametrizes all spacelike and null planes in $\mathbb{R}^{2,1}$. Adorned with an appropriate geometric structure, the space of such planes is known in the literature as co-Minkowski space [8] or half-pipe geometry [6]. Of course, we could just as well think of it as the space of all future-complete half-spaces in $\mathbb{R}^{2,1}$ with spacelike or null boundary.

For our purposes, we are concerned only with the topology and convexity of this space. Recall that if $f$ is a function on $\mathbb{R}^{2}$ valued in $\mathbb{R} \cup\{+\infty\}$ and not identically equal to $+\infty$, the Legendre transform of $f$ is the function $f^{*}: \mathbb{R}^{2} \rightarrow \mathbb{R} \cup\{+\infty\}$ defined by

$$
f^{*}(\mathrm{y})=\sup _{\mathbf{x} \in \mathbb{R}^{2}}(\mathrm{y} \cdot \mathrm{x}-f(\mathrm{x}))
$$

It follows from the definitions of support function and Legendre transform that, if an achronal $\Sigma$ is the graph of some function $f: \mathbb{R}^{2} \rightarrow \mathbb{R}$, then its support function $u_{\Sigma}$ equals the Legendre transform $f^{*}$ restricted to $\overline{\mathbb{D}}$. Moreover, $f^{*}$ is $+\infty$ outside $\overline{\mathbb{D}}$. 
Proposition 2.3 [16, Cor 12.2.1] The Legendre transform gives an involutive one-toone correspondence between proper closed convex functions on $\mathbb{R}^{2}$.

Restricting to functions on the closed disk and associating an entire achronal surface (which is the graph of a 1-Lipschitz function) with the function of which it is a graph, an immediate corollary is the following version of convex duality:

Proposition 2.4 The Legendre transform gives an involutive bijection between entire convex achronal surfaces and proper closed convex functions on $\overline{\mathbb{D}}$.

Now we concentrate on the support function of a regular domain. Recall that a regular domain $\mathcal{D}$ is an open domain which can be written as the intersection of the futures of a family $\mathcal{F}$ of at least two nonparallel null planes in $\mathbb{R}^{2,1}$. Thinking of $\mathbb{D} \times \mathbb{R}$ as the space of null or spacelike planes in $\mathbb{R}^{2,1}$, we view $\mathcal{F}$ as a subset of $\partial \mathbb{D} \times \mathbb{R}$. The family $\mathcal{F}$ is not unique - for instance, we may add to the family a null plane parallel to and lying below a plane already in $\mathcal{F}$ without changing the domain $\mathcal{D}$. However, the union of defining families is still a defining family, so given a regular domain $\mathcal{D}$ we may consider the maximal family $\mathcal{F}_{\mathcal{D}}$ of defining planes. Since $\mathcal{D}$ is assumed to be open, a limit of planes disjoint from $\mathcal{D}$ is still disjoint from $\mathcal{D}$, so since $\mathcal{F}_{\mathcal{D}}$ is maximal it must be closed as a subset of $\partial \mathbb{D} \times \mathbb{R}$. Since it is also upward-closed, $\mathcal{F}_{\mathcal{D}}$ is the supergraph of a closed function $\varphi_{\mathcal{D}}$ on $\partial \mathbb{D}$. Note that $\varphi_{\mathcal{D}}$ is finite at at least two points because the set $\mathcal{F}_{\mathcal{D}}$ by assumption contains at least two non-parallel planes. As a consequence we obtain the following proposition:

Proposition 2.5 The assignment $\mathcal{D} \mapsto \varphi_{\mathcal{D}}$ is a bijection between the set of regular domains and the set of proper closed functions on the circle which are finite at at least two points.

We will use the notation $\mathcal{D}_{\varphi}$ to represent the domain corresponding to $\varphi$.

Another important notion of convex geometry is the convex envelope.

Definition 2.6 If $f$ is any function on $\mathbb{R}^{2}$ valued in $\mathbb{R} \cup\{+\infty\}$, the convex envelope $\operatorname{conv}(f)$ is the function whose supergraph is the closure of the convex hull of the supergraph of $f$.

Equivalently [16, Cor 12.1.1], $\operatorname{conv}(f)$ can be equivalently expressed as the supremum of affine functions less than or equal to $f$ :

$$
\operatorname{conv}(f)(\mathbf{x})=\sup \left\{v(\mathbf{x}) \mid v: \mathbb{R}^{2} \rightarrow \mathbb{R} \text { is affine, } v \leq f\right\} .
$$

Proposition 2.7 Let $\mathcal{D}$ be a regular domain. Then the support function $u_{\mathcal{D}}$ is equal to $\operatorname{conv}\left(\varphi_{\mathcal{D}}\right)$. Moreover $u_{\mathcal{D}}$ restricted to the unit circle is equal to $\varphi_{\mathcal{D}}$ and if $\varphi_{\mathcal{D}}$ is infinite on an open arc with endpoints $\xi_{1}$ and $\xi_{2}$, then $u_{\mathcal{D}}$ restricted to the chord $\left[\xi_{1}, \xi_{2}\right]$ is the convex envelope of $\left.\varphi_{\mathcal{D}}\right|_{\left\{\xi_{1}, \xi_{2}\right\}}$.

Let us write $\varphi=\varphi_{\mathcal{D}}$. The last property of $u_{\mathcal{D}}$ says that $u_{\mathcal{D}}$ restricted to the open chord $\left(\xi_{1}, \xi_{2}\right)$ is infinite if either $\varphi\left(\xi_{1}\right)$ or $\varphi\left(\xi_{2}\right)$ are infinite, and otherwise is the unique affine function interpolating $\varphi\left(\xi_{1}\right)$ and $\varphi\left(\xi_{2}\right)$. Note that this also implies that the essential support of $\operatorname{conv}(\varphi)$ is the convex hull of the essential support of $\varphi$. 
Proof By construction, $\mathcal{D}$ is the strict supergraph of the Legendre transform $\varphi^{*}$. Since the support function of $\mathcal{D}$ is the same as the support function of its closure and the support function is the restriction of the Legendre transform to the disk, $u_{\mathcal{D}}=\varphi^{* *}$. By [16, Thm 12.2], $\varphi^{* *}=\operatorname{conv}(\varphi)$.

We now show that as long as $\varphi$ is lower semi-continuous, $\operatorname{conv}(\varphi)$ restricted to the unit circle is equal to $\varphi$. Let $\varphi^{+}$be the supergraph of $\varphi$. By assumption it is closed, and the first thing we need to show is that its convex hull is still closed. According to [16, Cor 17.2], if $S$ is a bounded set of points in $\mathbb{R}^{n}$, then $\operatorname{cl}(\operatorname{conv}(S))=\operatorname{conv}(\operatorname{cl}(S))$. We would like to apply this theorem with $S=\varphi^{+}$, but since it is not bounded so we need a slightly generalized theorem. If we include $\mathbb{R}^{2} \times \mathbb{R}$ into $\mathbb{R P}^{3}$, then the union of $\varphi^{+}$with the point at $z=+\infty$ is still closed, and after a projective transformation it is bounded in $\mathbb{R}^{2} \times \mathbb{R}$. Applying the closure theorem to this transformed set and then transforming back, we conclude that the convex hull of $\varphi^{+}$is closed.

Therefore, the supergraph of $\operatorname{conv}(\varphi)$ is actually the convex hull of $\varphi^{+}$, not just its closure. Hence any point in the supergraph of $u_{\mathcal{D}}$ is a convex linear combination of finitely many points in $\varphi^{+}$. If $\varphi$ is supported on only one side of a line $L$, then each point in the graph of $\left.u_{\mathcal{D}}\right|_{L}$ is a convex linear combination of only those points in $\left.\varphi^{+}\right|_{L}$.

Applying this observation to the case where $L$ is tangent to the unit circle, we see that $u_{\mathcal{D}}$ restricted to the unit circle is equal to $\varphi$. Applying the observation to the case where $L$ contains a chord $\left[\xi_{1}, \xi_{2}\right]$ as in the statement of the proposition, we conclude that $u_{\mathcal{D}}$ restricted to $\left[\xi_{1}, \xi_{2}\right]$ is the convex envelope of $\left.\varphi\right|_{\left\{\xi_{1}, \xi_{2}\right\}}$.

\subsection{A Dirichlet-type problem}

In this section, we characterize the support function of an entire future-convex spacelike surface with prescribed Gaussian curvature, and show that the problem of finding such a surface in a given domain of dependence is dual to a Dirichlet-like problem for the support function.

In the following, let $\Sigma$ be an entire future-convex spacelike surface in $\mathbb{R}^{2,1}$. By Corollary 1.17 , the domain of dependence of $\Sigma$ is the intersection of the futurecomplete open null half-spaces containing it. Hence we have the following lemma:

Lemma 2.8 Let $\Sigma$ be an entire spacelike future-convex surface with domain of dependence $\mathcal{D}$. Let $u_{\Sigma}$ be the support function of $\Sigma$ and let $u_{\mathcal{D}}$ be the support function of $\mathcal{D}$. Then $u_{\Sigma}$ and $u_{\mathcal{D}}$ coincide on the unit circle.

The fact that $\Sigma$ is entire gives another restriction on its support function $u_{\Sigma}$. In order to describe this condition, we first define the domain of support of a proper closed convex function to be the interior of its essential domain, i.e. the largest open set on which $u$ is finite. We will use $\Omega_{u}$ to denote the domain of support of $u$. Since $u$ is convex, so is its essential domain, which implies that the essential domain of $u$ is either contained in a line or has nonempty interior. Setting the first possibility aside for the moment, assume that $\Omega_{u}$ is nonempty. The essential domain of $u$ is contained in $\bar{\Omega}_{u}$ and since the function $u$ is closed its values on the boundary of $\Omega_{u}$ are uniquely determined by its restriction to $\Omega_{u}$.

Now we may make the definition: 
Definition 2.9 Let $u$ be a proper closed convex function on $\mathbb{R}^{2}$ such that $\Omega_{u}$ is nonempty and bounded and $u$ is differentiable throughout $\Omega_{u}$. The function $u$ is called gradient surjective if its gradient map $D u: \Omega_{u} \rightarrow \mathbb{R}^{2}$ is surjective.

By a special case of [16, Thm 26.3], a function $u$ is gradient surjective if and only if its Legendre transform $u^{*}$ is entire and strictly convex. By convex duality (Proposition 2.4), this implies:

Lemma 2.10 The support function of a strictly future-convex entire spacelike surface is gradient surjective.

Applying a variant of the same theorem [16, Thm. 26.3] to $u_{\Sigma}^{*}$, we also see that if $\Sigma$ is $C^{1}$ as well as being strictly convex, then the gradient map $D u_{\Sigma}$ is injective as well, so by invariance of domain it gives a homeomorphism from $\Omega_{u}$ to $\mathbb{R}^{2}$. We remark that this gradient is related to the inverse of the Gauss map of $\Sigma$. Namely, let us denote by $\pi: \mathbb{H}^{2} \rightarrow \mathbb{D}$ the radial projection from the hyperboloid to the disc at height one, namely

$$
\pi\left(y_{1}, y_{2}, y_{3}\right)=\left(\frac{y_{1}}{y_{3}}, \frac{y_{2}}{y_{3}}\right),
$$

which gives an identification of the hyperboloid model of Example 1.3 with the Klein model of the hyperbolic plane. Then the composition $\pi \circ G_{\Sigma}$ of the projection with the Gauss map is inverse to the map

$$
\mathrm{y} \mapsto\left(D u_{\Sigma}(\mathrm{y}), u_{\Sigma}^{*}\left(D u_{\Sigma}(\mathrm{y})\right)\right)
$$

as maps between $\Sigma$ and $\Omega_{u_{\Sigma}}$ [2, Lemma 2.15]. Moreover, we note that if $\Sigma$ is convex then

$$
u_{\Sigma}\left(\pi \circ G_{\Sigma}(\boldsymbol{p})\right)=\left\langle\boldsymbol{p},\left(\pi \circ G_{\Sigma}(\boldsymbol{p}), 1\right)\right\rangle
$$

and if $\Sigma$ is entire then $u_{\Sigma}(\mathrm{y})=+\infty$ if $\mathrm{y} \notin \overline{\pi \circ G_{\Sigma}(\Sigma)}$.

We now provide a formula which relates the Gaussian curvature of a $C^{2}$ strictly convex spacelike surface $\Sigma$ to the support function $u_{\Sigma}$.

Lemma 2.11 [15] Let $u_{\Sigma}: \mathbb{D} \rightarrow \mathbb{R}$ be the support function of a future-convex $C^{2}$ spacelike embedded surface $\Sigma$ in $\mathbb{R}^{2,1}$. Then $u_{\Sigma}$ satisfies

$$
\operatorname{det} D^{2} u_{\Sigma}(x)=\frac{1}{\psi(x)}\left(1-|x|^{2}\right)^{-2} .
$$

for every $x \in \Omega_{u_{\Sigma}}$, where $\psi=\kappa_{\Sigma} \circ\left(\pi \circ G_{\Sigma}\right)^{-1}$ is the curvature function, and $\kappa_{\Sigma}=\operatorname{det} B$ is the Gaussian curvature of $\Sigma$.

In particular, if $\Sigma$ is a future-convex surface of constant Gaussian curvature det $B \equiv$ $K>0$ (as in Definition 1.2), then on the image of the Gauss map $u_{\Sigma}$ satisfies:

$$
\operatorname{det} D^{2} u_{\Sigma}(\mathbf{x})=\frac{1}{K}\left(1-|\mathbf{x}|^{2}\right)^{-2} .
$$


At last we are ready to translate our original problem of prescribed Gaussian curvature into a Dirichlet-like problem for the support function.

Definition 2.12 Let $\varphi: \partial \mathbb{D} \rightarrow \mathbb{R} \cup\{+\infty\}$ be lower semicontinuous and let $\psi: \mathbb{D} \rightarrow$ $\mathbb{R}$. We say that a proper closed convex function $u: \mathbb{D} \rightarrow \mathbb{R} \cup\{+\infty\}$ is a solution of the Minkowski problem with curvature function $\psi$ and boundary data $\varphi$ if

- $u$ is equal to $\varphi$ when restricted to $\partial \mathbb{D}$,

- $u \in C^{2}\left(\Omega_{u}\right)$ and solves the equation

$$
\operatorname{det} D^{2} u(\mathrm{x})=\frac{1}{\psi(\mathrm{x})}\left(1-|\mathrm{x}|^{2}\right)^{-2},
$$

on the domain $\Omega_{u}$.

With this definition, we obtain an equivalent formulation of the Minkowski problem, as stated in Sect. 1.5:

Proposition 2.13 Given any $\varphi: \partial \mathbb{D} \rightarrow \mathbb{R} \cup\{+\infty\}$ lower semicontinuous and finite at at least 3 points, and any $\psi: \mathbb{D} \rightarrow \mathbb{R}$ smooth, $u$ is a gradient-surjective solution of the Minkowski problem with data $\varphi$ and $\psi$ if and only if $u$ is the support function of an entire spacelike surface $\Sigma$ such that $\mathcal{D}_{\Sigma}=\mathcal{D}_{\varphi}$ and $\psi=\kappa_{\Sigma} \circ\left(\pi \circ G_{\Sigma}\right)^{-1}$.

\subsection{Gaussian curvature and examples}

Let us now give two first basic explicit examples:

Example 2.14 The hyperboloid Hyp (see Example 1.3), rescaled by a factor $1 / \sqrt{K}$, is an entire strictly future-convex surface (which we denote $\mathrm{Hyp}^{K}$ ) of constant Gaussian curvature $K$. In fact, it can be checked directly that (if $\boldsymbol{n}$ is the future unit normal field) its shape operator is $B=D \boldsymbol{n}=\sqrt{K} \mathbb{1}$, where $\mathbb{1}$ is the identity operator. Such surface is invariant by the group of linear isometries $\mathrm{SO}_{0}(2,1)$. Its support function is:

$$
u_{\mathrm{Hyp}^{K}}(\mathrm{x})=-\frac{1}{\sqrt{K}} \sqrt{1-|\mathrm{x}|^{2}},
$$

which is a solution of Eq. (9). Observe that $u_{\mathrm{Hyp}^{K}}$ is finite on the whole disk and $u_{\mathrm{Hyp}^{K}}=0$ on $\partial \mathbb{D}$.

Example 2.15 We have introduced in Example 1.4 the trough:

$$
T:=\left\{x \in \mathbb{R}^{2,1}: x_{2}^{2}-x_{3}^{2}=-1, x_{3}>0\right\},
$$

Its support function, at any point $\mathrm{x}=(x, y)$, is:

$$
u_{T}(x, y)= \begin{cases}-\sqrt{1-y^{2}} & \text { if } x=0 \text { and } y \in[-1,1] \\ +\infty & \text { otherwise }\end{cases}
$$

We remark that the trough is convex but not strictly convex and has Gaussian curvature 0 . The essential support of $u_{T}$ is a segment. 


\section{Tools from Monge-Ampère equations}

In order to prove the existence and uniqueness of entire surfaces of prescribed curvature, we will construct solutions of Eq. (8). For this purpose, we will need several tools from the classical theory of Monge-Ampère equations-in particular, the notion of generalized solution, the maximum principle, and some results of existence and regularity. The purpose of this section is to collect the necessary tools and prove a generalized maximum principle for Monge-Ampère equations.

\subsection{Generalized solutions}

Given a convex function $u: \Omega \rightarrow \mathbb{R}$ for $\Omega$ a convex domain in $\mathbb{R}^{2}$, we define the subdifferential of $u$ as the set-valued function $\partial_{u}$ whose value at a point $\mathrm{x} \in \Omega$ is:

$\partial_{u}(\mathrm{x})=\{D v \mid v$ affine; $\operatorname{graph}(v)$ is a support plane for $\operatorname{graph}(u) ;(\mathrm{x}, u(\mathrm{x})) \in \operatorname{graph}(v)\}$.

In general $\partial_{u}(\mathrm{x})$ is a convex set. If $u$ is differentiable at $\mathrm{x}$, then $\partial_{u}(\mathrm{x})=\{D u(\mathrm{x})\}$. We define the Monge-Ampère measure on the collection of Borel subsets $\omega$ of $\mathbb{R}^{2}$ :

$$
M A_{u}(\omega)=\mathcal{L}\left(\partial_{u}(\omega)\right)
$$

where $\mathcal{L}$ denotes the Lebesgue measure on $\mathbb{R}^{2}$.

Lemma 3.1 ([19, Lemma 2.3]) If $u$ is a $C^{2}$ convex function, then

$$
M A_{u}(\omega)=\mathcal{L}(D u(\omega))=\int_{\omega}\left(\operatorname{det} D^{2} u\right) d \mathcal{L} .
$$

Definition 3.2 Given a nonnegative measure $v$ on $\Omega$, we say a convex function $u$ : $\Omega \rightarrow \mathbb{R}$ is a generalized solution to the Monge-Ampère equation

$$
\operatorname{det} D^{2} u=v
$$

if $M A_{u}(\omega)=v(\omega)$ for all Borel subsets $\omega$. In particular, given an integrable function $f: \Omega \rightarrow \mathbb{R}, u$ is a generalized solution to the equation $\operatorname{det} D^{2} u=f$ if and only if, for all $\omega$,

$$
M A_{u}(\omega)=\int_{\omega} f d \mathcal{L}
$$

We collect here, without proofs, some facts which will be used in the following. Unless explicitly stated, the results hold in $\mathbb{R}^{n}$, although we are only interested in $n=2$. 


\subsection{Stability and comparison principle}

Let us start by the following important lemma, which concerns the continuity of the Monge-Ampère measure.

Lemma 3.3 ([19, Lemma 2.2]) Let $u_{n}$ be a sequence of convex functions on a convex domain $\Omega$. If $u_{n}$ converges uniformly on compact sets to $u_{\infty}$, then the Monge-Ampère measures $M A_{u_{n}}$ converge weakly to $M A_{u_{\infty}}$.

Second, the following comparison principle is the key ingredient, for instance, for every result of uniqueness.

Theorem 3.4 (Maximum principle, $[11,19]$ ) Given a bounded convex domain $\Omega$ and two convex functions $u_{+}, u_{-} \in C^{0}(\bar{\Omega})$, if $M A_{u_{+}}(\omega) \leq M A_{u_{-}}(\omega)$ for every Borel subset $\omega$, then

$$
\min _{\bar{\Omega}}\left(u_{+}-u_{-}\right)=\min _{\partial \Omega}\left(u_{+}-u_{-}\right) .
$$

The following is a direct consequence.

Corollary 3.5 (Comparison principle) Given a bounded convex domain $\Omega$ and two convex functions $u_{+}, u_{-} \in C^{0}(\bar{\Omega})$, if $u_{+} \geq u_{-}$on $\partial \Omega$ and $M A_{u_{+}}(\omega) \leq M A_{u_{-}}(\omega)$ for every Borel subset $\omega$, then $u_{+} \geq u_{-}$on $\Omega$.

In particular, we have the following result of uniqueness.

Corollary 3.6 Given two generalized solutions $u_{1}, u_{2} \in C^{0}(\bar{\Omega})$ to the Monge-Ampère equation det $D^{2} u=v$ on a bounded convex domain $\Omega$, if $u_{1} \equiv u_{2}$ on $\partial \Omega$, then $u_{1} \equiv u_{2}$ on $\Omega$.

\subsection{Existence and regularity}

The following is a classical result of existence for the Dirichlet problem for MongeAmpère equations.

Theorem 3.7 (Dirichlet problem, [11, Theorem 1.6.2]) Let $\Omega$ be a bounded strictly convex domain. Given any continuous function $g: \partial \Omega \rightarrow \mathbb{R}$ and any Borel measure $v$ with $v(\Omega)<+\infty$, there exists a generalized solution $u \in C^{0}(\bar{\Omega})$ of the problem

$$
\left\{\begin{array}{l}
\operatorname{det} D^{2} u=v \quad \text { in } \Omega \\
\left.u\right|_{\partial \Omega}=g
\end{array}\right.
$$

We remark here that Theorem 3.7 does not apply directly to Equation (8), since in that case the hypothesis of finite total measure is not satisfied. Moreover, the boundary value will not be continuous in the general problem we consider. We also have the following important regularity property: 
Theorem 3.8 ([19, Theorem 3.1]) Let $u$ be a strictly convex generalized solution to $\operatorname{det} D^{2} u=f$ on a bounded convex domain $\Omega$ with smooth boundary. If $f>0$ and $f$ is smooth, then $u$ is smooth.

The following property will be used repeatedly in the paper, and is a peculiar property of dimension $n=2$.

Theorem 3.9 (Aleksandrov-Heinz, [19, Remark 3.2]) Let $f$ be a positive function and let $u$ be a generalized solution of the Monge-Ampère equation $\operatorname{det} D^{2} u=f$ on a domain $\Omega \subset \mathbb{R}^{2}$. Then u is strictly convex.

\subsection{A generalized comparison principle}

In this section we will prove a version of the maximum principle (Theorem 3.4) which we can apply to functions valued in $\mathbb{R} \cup\{+\infty\}$ which satisfy a Monge-Ampère equation on their domain of support. The following definition generalizes Definition 2.9.

Definition 3.10 A closed convex function $u$ on $\mathbb{R}^{2}$ taking values in $\mathbb{R} \cup\{+\infty\}$ is gradient-surjective if the sub-differential gives a surjective set-valued map from the interior of its essential domain to $\mathbb{R}^{2}$.

Proposition 3.11 (Generalized comparison principle) Suppose $\Omega$ is a convex bounded domain, $u_{+}: \bar{\Omega} \rightarrow \mathbb{R} \cup\{+\infty\}$ is a closed convex function, and $u_{-} \in C^{0}(\bar{\Omega})$ is convex. If $u_{+}$is gradient-surjective and $M A_{u_{+}}(\omega) \leq M A_{u_{-}}(\omega)$ for every Borel subset $\omega \subset \Omega_{u_{+}}$, then

$$
\min _{\bar{\Omega}}\left(u_{+}-u_{-}\right)=\min _{\partial \Omega}\left(u_{+}-u_{-}\right)
$$

Proof Under the assumptions, the function $u_{+}-u_{-}$is lower-semicontinuous on $\bar{\Omega}$, so it attains its minimum value at some point $x_{0} \in \bar{\Omega}$. We first show that $x_{0} \notin \partial \Omega_{u_{+}} \backslash \partial \Omega$.

Indeed, suppose otherwise. Let $p \in \partial_{u_{-}}\left(\mathrm{x}_{0}\right)$, and let $l(\mathrm{x})=u_{-}\left(\mathrm{x}_{0}\right)+p \cdot\left(\mathrm{x}-\mathrm{x}_{0}\right)$ be the corresponding affine support. Since $u_{+}$is convex, the set $\Omega_{u_{+}}$is convex, so it has a supporting hyperplane at $\mathrm{x}_{0}$. Let $q$ be the outward normal vector to such a hyperplane, so that the linear function $m(\mathrm{x})=q \cdot\left(\mathrm{x}-\mathrm{x}_{0}\right)$ is negative on $\Omega_{u_{+}}$. Let $\tilde{p}=p+q$. Since $l$ is a support for $u_{-}$, for any $\mathrm{x} \in \Omega_{u_{+}}$, we have $l(x)<u_{-}(x)$, in other words

$$
\tilde{p} \cdot\left(\mathrm{x}-\mathrm{x}_{0}\right)<u_{-}(\mathrm{x})-u_{-}\left(\mathrm{x}_{0}\right) .
$$

Now we use the property that $u_{+}$is gradient-surjective to find a point $x_{1} \in \Omega_{u_{+}}$for which $\tilde{p} \in \partial_{u_{+}}\left(\mathrm{x}_{1}\right)$. Let $\tilde{l}(\mathrm{x})$ be the corresponding affine support for $u_{+}$at $\mathrm{x}_{1}$, that is

$$
\tilde{l}(\mathrm{x})=u_{+}\left(\mathrm{x}_{1}\right)+\tilde{p} \cdot\left(\mathrm{x}-\mathrm{x}_{1}\right) .
$$

Using $\tilde{l}\left(\mathrm{x}_{0}\right) \leq u_{+}\left(\mathrm{x}_{0}\right)$, we have

$$
u_{+}\left(\mathrm{x}_{1}\right)-u_{+}\left(\mathrm{x}_{0}\right) \leq \tilde{p} \cdot\left(\mathrm{x}_{1}-\mathrm{x}_{0}\right) .
$$


Since $u_{+}-u_{-}$is minimized at $\mathrm{x}_{0}$, we have

$$
u_{+}\left(\mathrm{x}_{0}\right)-u_{-}\left(\mathrm{x}_{0}\right) \leq u_{+}\left(\mathrm{x}_{1}\right)-u_{-}\left(\mathrm{x}_{1}\right) .
$$

Putting these inequalities together gives

$$
\tilde{p} \cdot\left(\mathrm{x}_{1}-\mathrm{x}_{0}\right)<u_{-}\left(\mathrm{x}_{1}\right)-u_{-}\left(\mathrm{x}_{0}\right) \leq u_{+}\left(\mathrm{x}_{1}\right)-u_{+}\left(\mathrm{x}_{0}\right) \leq \tilde{p} \cdot\left(\mathrm{x}_{1}-\mathrm{x}_{0}\right)
$$

which is a contradiction. We conclude that $\mathrm{x}_{0} \notin \partial \Omega_{u_{+}} \backslash \partial \Omega$.

The rest of the argument is essentially the proof of the standard comparison principle (following [11, Theorem 1.4.6]). Suppose that $\mathrm{x}_{0} \in \Omega_{u_{+}}$and also for the sake of contradiction that

$$
u_{+}\left(\mathrm{x}_{0}\right)-u_{-}\left(\mathrm{x}_{0}\right)<\min _{\partial \Omega}\left(u_{+}-u_{-}\right)
$$

Then it follows also that

$$
u_{+}\left(x_{0}\right)-u_{-}\left(x_{0}\right)<\min _{\partial \Omega_{u_{+}}}\left(u_{+}-u_{-}\right),
$$

since otherwise the minimum would be attained on $\partial \Omega_{u_{+}} \backslash \partial \Omega$. By adding a suitable constant to $u_{-}$, we may arrange that

$$
u_{+}\left(\mathrm{x}_{0}\right)-u_{-}\left(\mathrm{x}_{0}\right)<0<\min _{\partial \Omega_{u_{+}}}\left(u_{+}-u_{-}\right) .
$$

By replacing $u_{-}$with $u_{-}+\delta\left|\mathrm{x}-\mathrm{x}_{0}\right|^{2}$ for small enough $\delta$, we can preserve these inequalities and also arrange that $M A_{u_{+}}(\omega)<M A_{u_{-}}(\omega)$ with strict inequality.

Let $U=\left\{\mathrm{x} \mid u_{+}(\mathrm{x})-u_{-}(\mathrm{x})<0\right\}$. A priori, since $u_{+}$is only semicontinuous, $U$ need not be open; however, by arrangement $U \subset \Omega_{u_{+}}$, and $u_{+}$is continuous on $\Omega_{u_{+}}$, so indeed $U$ is open. In fact, the set $\left\{\mathrm{x} \mid u_{+}(\mathrm{x})-u_{-}(\mathrm{x}) \leq 0\right\}$ is closed and contained in $\Omega_{u_{+}}$, so $U$ is compactly contained in $\Omega_{u_{+}}$, and $u_{+}$is continuous on $\bar{U}$. Hence, $u_{+}=u_{-}$on the boundary of $U$, with $u_{+}<u_{-}$on the interior. It follows that $\partial_{u_{-}}(U) \subset \partial_{u_{+}}(U)$, which contradicts the strict inequality $M A_{u_{+}}(U)<M A_{u_{-}}(U)$.

The following is a straightforward consequence of the generalized comparison principle.

Proposition 3.12 Let $\Omega$ be a convex domain. Suppose that $u_{+}, v: \bar{\Omega} \rightarrow \mathbb{R} \cup\{+\infty\}$ are closed convex functions with $u_{+}$gradient-surjective, $v \in C^{0}(\bar{\Omega})$, and $M A_{u_{+}}(\omega) \leq$ $M A_{v}(\omega)$ for every Borel subset $\omega \subset \Omega_{u_{+}}$. Suppose furthermore that $v(\xi) \leq 0$ at every point $\xi \in \partial \Omega$ for which $u_{+}(\xi)<+\infty$. Then

$$
u_{+} \geq \operatorname{conv}\left(\left.u_{+}\right|_{\partial \Omega}\right)+v .
$$

Proof Set $\varphi=\left.u_{+}\right|_{\partial \Omega}$. By the remark following Definition 2.6, it is enough to show that $u_{+} \geq l+v$ for every affine function $l$ on $\bar{\Omega}$ with $\left.l\right|_{\partial \Omega} \leq \varphi$. By the assumption 
on $v$, the restriction of $l+v$ to $\partial \Omega$ is less than or equal to $\varphi$, and its Monge-Ampère measure coincides with that of $v$. Hence we may apply the generalized comparison principle to conclude $u_{+} \geq l+v$.

\section{Gauss map and minimal Lagrangian maps}

In this section, we will study some properties of the Gauss map and the support function of future-convex entire surfaces with Gaussian curvature bounded from above and below by positive constants. We thus prove Theorem 4.4 , which is a refined version of Theorem E. We will then study the relation with minimal Lagrangian maps with values in the hyperbolic plane, and derive Corollary $\mathrm{G}$ as a consequence.

\subsection{Classical barriers}

We give here the construction of some explicit surfaces of constant Gaussian curvature. Besides being examples of the theory previously explained, Example 4.3 will serve as a barrier in the proof of Theorem 4.4 below.

These surfaces are obtained as surfaces of revolution, that is, they are invariant under a 1-parameter group of hyperbolic isometries in $\operatorname{SO}(2,1)<\operatorname{Isom}\left(\mathbb{R}^{2,1}\right)$. Surfaces of this form were studied in [12], where the first examples of non-standard isometric embeddings of $\mathbb{H}^{2}$ in $\mathbb{R}^{2,1}$ were provided. Up to conjugation, we can assume the 1-parameter group has the form

$$
\left\{\left(\begin{array}{ccc}
1 & 0 & 0 \\
0 & \cosh (s) & \sinh (s) \\
0 & \sinh (s) & \cosh (s)
\end{array}\right) \mid s \in \mathbb{R}\right\} .
$$

Hence we consider surfaces $\Sigma$ parameterized by

$$
(t, s) \mapsto\left\{\begin{array}{l}
x_{1}(t, s)=g(t) \\
x_{2}(t, s)=\sinh (s) r(t) \\
x_{3}(t, s)=\cosh (s) r(t)
\end{array}\right.
$$

That is, we apply the 1-parameter hyperbolic group to the planar curve $(g(t), 0, r(t))$. Following [12], one can assume that

$$
g^{\prime}(t)^{2}-r^{\prime}(t)^{2}=1
$$

which means that, for $s=s_{0}$ fixed, the planar curve $\Sigma \cap\left\{x_{2} \cosh \left(s_{0}\right)=x_{3} \sinh \left(s_{0}\right)\right\}$ is parameterized by arclength.

Remark 4.1 Viewing the space $\overline{\mathbb{D}} \times \mathbb{R}$ as the space of achronal planes in $\mathbb{R}^{2,1}$, it is straightforward to write down the action of the 1-parameter group of Eq. (11) on this space. Using the fact that if $\Sigma$ is invariant under this group then so must be the graph 
of its support function $u_{\Sigma}$ in $\overline{\mathbb{D}} \times \mathbb{R}$, it can be shown that $u_{\Sigma}$ satisfies the following invariance (compare Equation (18)):

$$
u_{\Sigma}(x, y)=\sqrt{1-y^{2}} \cdot u_{\Sigma}\left(\frac{x}{\sqrt{1-y^{2}}}, 0\right) .
$$

As a consequence, if $\xi=(x, y) \in \partial \mathbb{D}$ so that $x^{2}+y^{2}=1$, then

$$
u_{\Sigma}(\xi)=|x| \cdot u_{\Sigma}\left(\frac{x}{|x|}, 0\right)=\left\{\begin{array}{ll}
x \cdot u_{\Sigma}(1,0) & \text { if } x \geq 0 \\
-x \cdot u_{\Sigma}(-1,0) & \text { if } x<0
\end{array} .\right.
$$

This function is affine on both half-planes $x \geq 0$ and $x \leq 0$. If $u_{\Sigma}(1,0)+u_{\Sigma}(-1,0)=$ 0 , then the two affine functions agree, and $\left.u_{\Sigma}\right|_{\partial \mathbb{D}}$ coincides with support function of the future of a point. If $u_{\Sigma}(1,0)+u_{\Sigma}(-1,0)>0$ then the two affine functions meet at a convex angle, and $\left.u_{\Sigma}\right|_{\partial \mathbb{D}}$ coincides with the support function of the future of the segment with end points $\left(u_{\Sigma}(-1,0), 0,0\right)$ and $\left(u_{\Sigma}(1,0), 0,0\right)$. If $u_{\Sigma}(1,0)+$ $u_{\Sigma}(-1,0)<0$ then $\left.u_{\Sigma}\right|_{\partial \mathbb{D}}$ coincides with the support function of the future of a hyperbola given as the intersection of the null cones of two points.

Example 4.2 (Entire CGC surfaces with surjective Gauss map) The Gaussian curvature of the surface parametrized by (12) assuming (13) is given by the simple formula $K(s, t)=r^{\prime \prime}(t) / r(t)$ [12, Equation 5]. For any $a>0$, we consider first the solution given by

$$
r(t)=a \cosh (t)
$$

which therefore has $g^{\prime}(t)=\sqrt{1+a^{2} \sinh ^{2}(t)}$. By choosing

$$
g(t)=\int_{0}^{t} \sqrt{1+a^{2} \sinh ^{2}(x)} d x
$$

the corresponding surface (say, $\Sigma_{a}$ ) is invariant by the reflection $\left(x_{1}, x_{2}, x_{3}\right) \mapsto$ $\left(-x_{1}, x_{2}, x_{3}\right)$. When written as a graph $\Sigma_{a}=\operatorname{graph}\left(f_{a}\right), f_{a}$ has therefore a minimum point at the origin. We remark that, when $a=1, \Sigma_{1}$ is the hyperboloid Hyp.

By multiplying $\Sigma_{a}$ by the factor $1 / \sqrt{K}$, one obtains analogously surfaces $\Sigma_{a}^{K}=$ $(1 / \sqrt{K}) \Sigma_{a}$ of constant Gaussian curvature $K$. To compute the support function $u_{\Sigma_{a}^{K}}$ of $\Sigma_{a}^{K}=\operatorname{graph}\left(f_{a}^{K}\right)$, we remark that $u_{\Sigma_{a}^{K}}(1,0)$ can be expressed as [4, Section 2.3]:

$$
u_{\Sigma_{a}^{K}}(1,0)=\lim _{x_{1} \rightarrow+\infty}\left(x_{1}-f_{a}^{K}\left(x_{1}, 0,0\right)\right)=\lim _{t \rightarrow+\infty}(g(t)-r(t))
$$

It can be thus shown that $F(a):=u_{\Sigma_{a}^{K}}(1,0)$ is finite for every $a$, is a decreasing function of $a$, and

$$
\lim _{a \rightarrow 0^{+}} F(a)=+\infty .
$$

Using Remark 4.1, we therefore have, for $\xi=(x, y) \in \partial \mathbb{D}$ : 


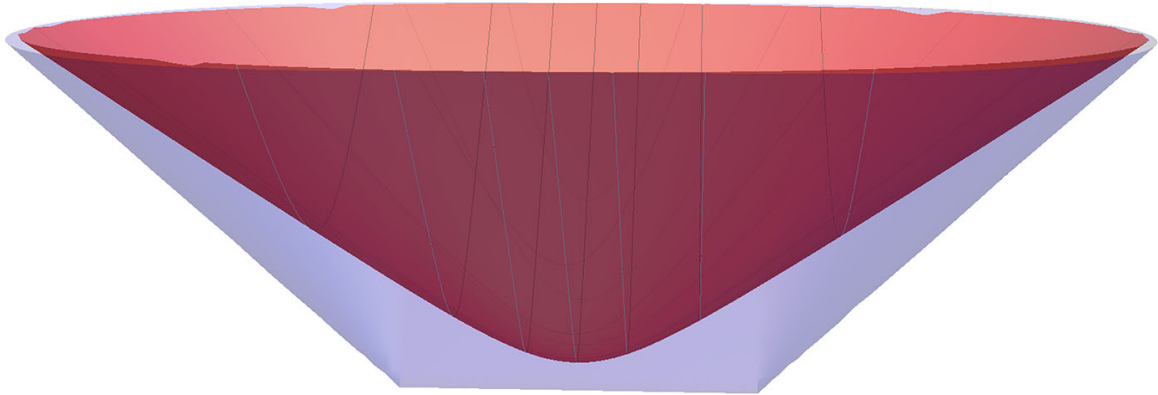

Fig. 3 The surface of revolution $\Sigma_{a}^{K}$, corresponding to the choice $r(t)=a \cosh (t)$ (here $K=1$ and $a=1 / 2$ ). The domain of dependence is the future of a spacelike segment

$$
u_{\Sigma_{a}^{K}}(\xi)=\frac{F(a)}{\sqrt{K}}|x|
$$

So, when $a \in(0,1)$, the domain of dependence of $\Sigma_{a}^{K}$ is the future of a segment. That is,

$$
\mathcal{D}_{\Sigma_{a}^{K}}=\bigcup_{x \in\left[-\frac{F(a)}{\sqrt{K}}, \frac{F(a)}{\sqrt{K}}\right]} I^{+}(x, 0,0) .
$$

See Fig. 3. From the expression (14) of Remark 4.1, we also see that $u_{\Sigma_{a}^{K}}$ is finite on $\overline{\mathbb{D}}$ and $u_{\Sigma_{a}^{K}} \in C^{0}(\overline{\mathbb{D}})$. Moreover, again from (14) we get:

$$
u_{\Sigma_{a}^{K}}(0, y)=\sqrt{1-y^{2}} u_{\Sigma_{a}^{K}}(0,0)=-\frac{a}{\sqrt{K}} \sqrt{1-y^{2}}
$$

which corresponds to the fact that $\Sigma_{a}^{K} \cap\left\{x_{1}=0\right\}$ is a hyperbola through the point $(0,0, a / \sqrt{K})$.

Example 4.3 (Entire CGC surfaces with Gauss map to a half-plane) Another useful family of surfaces, still studied in [12], is obtained by the choice $r(t)=e^{t}$. By writing the explicit expression of

$$
g(t)=\int_{0}^{t} \sqrt{1+r^{\prime}(x)^{2}} d x
$$

this gives:

$$
(t, s) \mapsto\left\{\begin{array}{l}
x_{1}(t, s)=\frac{1}{\sqrt{K}}\left(\sqrt{1+e^{2 t}}-\frac{1}{2} \log \left(\frac{\sqrt{1+e^{2 t}}+1}{\sqrt{1+e^{2 t}}-1}\right)\right) \\
x_{2}(t, s)=\frac{1}{\sqrt{K}} \sinh (s) e^{t} \\
x_{3}(t, s)=\frac{1}{\sqrt{K}} \cosh (s) e^{t}
\end{array}\right.
$$




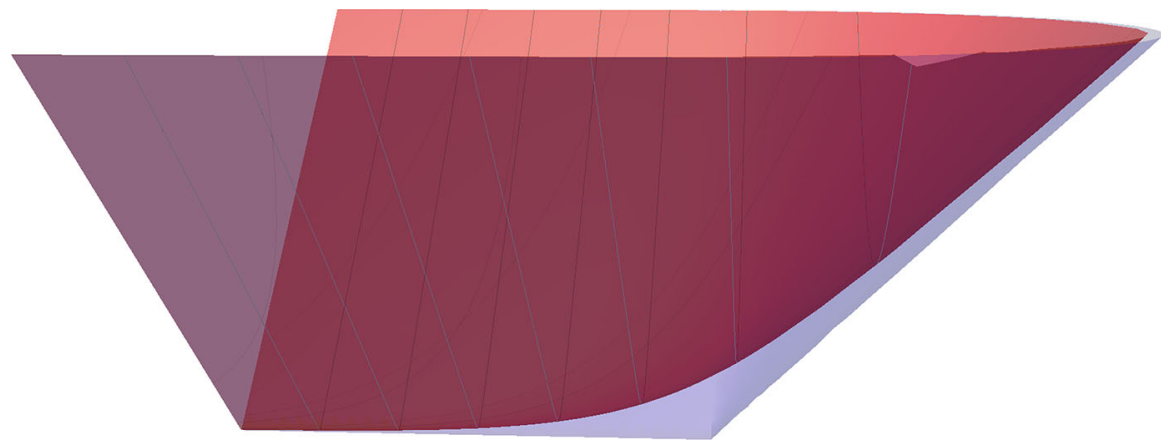

Fig. 4 The surface of revolution $\Sigma_{0}^{K}$, corresponding to the choice $r(t)=e^{t}$. (Here $K=1$.) The domain of dependence is the future of a half-line

Let us call $\Sigma_{0}^{K}$ such surface. See also Fig. 4. A direct computation, using Remark 4.1 shows that the corresponding support function is

$$
u_{\Sigma_{0}^{K}}(x, y)=\left\{\begin{array}{ll}
-\frac{1}{2 \sqrt{K}} x \log \left(\frac{1+\sqrt{1-\frac{x^{2}}{1-y^{2}}}}{1-\sqrt{1-\frac{x^{2}}{1-y^{2}}}}\right) & x \geq 0 \\
+\infty & x<0
\end{array} .\right.
$$

This is another solution of Eq. (9), which by direct inspection can be shown to be continuous on the closed half-space $\overline{\mathbb{D}}_{+}$, where $\mathbb{D}_{+}=\mathbb{D} \cap\{x>0\}$, and $u_{\Sigma_{0}^{K}}=0$ on $\partial \mathbb{D}_{+}$.

\subsection{Image of the Gauss map}

We will now prove the following theorem, which is a refined version of Theorem E, and gives a complete description of the image of the Gauss map of a CGC entire surface in $\mathbb{R}^{2,1}$.

Theorem 4.4 Let $\Sigma$ be an entire spacelike surface in $\mathbb{R}^{2,1}$ with Gaussian curvature bounded from above and below by positive constants. Let $u_{\Sigma}: \mathbb{D} \rightarrow \mathbb{R} \cup\{+\infty\}$ be the support function of $\Sigma$. Then

- The essential domain of $u_{\Sigma}$, i.e. the set on which $u_{\Sigma}$ is finite, coincides with the convex hull of $\left\{\xi \in \partial \mathbb{D} \mid u_{\Sigma}(\xi)<+\infty\right\}$.

- For every segment of $\partial \Omega_{\Sigma}$ with endpoints $\xi_{1}, \xi_{2} \in \partial \mathbb{D}, u_{\Sigma}$ restricted to the chord $\left[\xi_{1}, \xi_{2}\right]$ is the convex envelope of $\left.u_{\Sigma}\right|_{\left\{\xi_{1}, \xi_{2}\right\}}$.

The second bullet point means that if either $u_{\Sigma}\left(\xi_{1}\right)$ or $u_{\Sigma}\left(\xi_{2}\right)$ is infinite then $u_{\Sigma}$ is infinite on the open chord, and otherwise it is the unique affine function interpolating the values at the endpoints (compare the comment following Proposition 2.7). 
Proof of Theorem 4.4 Let $\mathcal{C}$ be the convex hull of $\left\{\xi \in \partial \mathbb{D}: u_{\Sigma}(\xi)<+\infty\right\}$. Let $K_{0}$ be a positive lower bound for the curvature of $\Sigma$. Let

$$
v(\mathrm{z})=-\frac{1}{\sqrt{K_{0}}} \sqrt{1-|\mathrm{z}|^{2}}
$$

be the support function of the hyperboloid $\operatorname{Hyp}^{K_{0}}$. Then $M A_{u_{\Sigma}}(\omega) \leq M A_{v}(\omega)$ for all Borel subsets $\omega \subset \Omega_{u_{\Sigma}}$ and $v$ is continuous on the closed disk and equal to 0 on the boundary. Hence by Proposition 3.12, we have $u_{\Sigma} \geq \operatorname{conv}\left(\left.u_{\Sigma}\right|_{\partial \mathbb{D}}\right)+v$. By the remark following Proposition 2.7 the essential support of $\operatorname{conv}\left(\left.u_{\Sigma}\right|_{\partial \mathbb{D}}\right)$ is equal to $\mathcal{C}$. Since $v$ is finite everywhere, this shows that $u_{\Sigma}$ is infinite at every point outside $\mathcal{C}$. Since $u_{\Sigma}$ is convex, so is its essential domain. Therefore the essential domain of $u_{\Sigma}$ is exactly $\mathcal{C}$. This proves the first bullet point as well as the second bullet point in the case where $u_{\Sigma}$ is infinite at either of the two endpoints $\xi_{i}$.

To complete the proof of the theorem, we need only consider the case where both $u_{\Sigma}\left(\xi_{1}\right)$ and $u_{\Sigma}\left(\xi_{2}\right)$ are finite for a segment $\left[\xi_{1}, \xi_{2}\right]$ of $\partial \Omega_{\Sigma}$. Up to composing $\Sigma$ with an isometry of $\mathbb{R}^{2,1}$, we can assume $\xi_{1}=(0,-1), \xi_{2}=(0,1)$ and that $\mathcal{C}$ is contained in $\{x \geq 0\}$. We will show that $u_{\Sigma}(0, y)=\operatorname{conv}\left(\left.u_{\Sigma}\right|_{\partial \mathbb{D}}\right)(0, y)$ for every $y \in[-1,1]$.

Let $\Sigma_{0}^{K_{0}}$ be the function constructed in Example 4.3, whose support function $u_{0}$ is a solution to the Monge Ampère equation (9) on the right half-disk $\mathbb{D}_{+}$with $\left.u_{0}\right|_{\partial \mathbb{D}_{+}}=0$. Let $A_{\epsilon}$ be the linear hyperbolic transformation of length $\epsilon$ with attracting fixed point $(-1,0)$ and repelling fixed point $(1,0)$. Let $u_{\epsilon}$ be the support function of $A_{\epsilon}\left(\Sigma_{0}^{K_{0}}\right)$. Explicitly, in coordinates $(x, y)$ on the disk [2, Lemma 3.4],

$u_{\epsilon}(x, y)=(\cosh (\epsilon)+x \sinh (\epsilon)) u_{0}\left(\frac{x \cosh (\epsilon)+\sinh (\epsilon)}{\cosh (\epsilon)+x \sinh (\epsilon)}, \frac{y}{\cosh (\epsilon)+x \sinh (\epsilon)}\right)$.

Observe that $u_{\epsilon}$ is equal to zero on the boundary of the half disk $\mathbb{D}_{+}^{\epsilon}$ bounded by the chord

$$
[(-\tanh (\epsilon), \operatorname{sech}(\epsilon)),(-\tanh (\epsilon),-\operatorname{sech}(\epsilon))]
$$

By Proposition 3.12 applied to $\mathbb{D}_{+}^{\epsilon}$, we have that

$$
u_{\Sigma} \geq \operatorname{conv}\left(\left.u_{\Sigma}\right|_{\partial \mathbb{D}_{+}^{\epsilon}}\right)+u_{\epsilon} \text { for all } \epsilon
$$

Since $u_{\Sigma}$ is equal to $+\infty$ on the left half-disk, in fact $\operatorname{conv}\left(\left.u_{\Sigma}\right|_{\partial \mathbb{D}_{+}^{\epsilon}}\right)=\operatorname{conv}\left(\left.u_{\Sigma}\right|_{\partial \mathbb{D}}\right)$. Now we take the limit as $\epsilon \rightarrow 0$ and use the continuity of $u_{0}$ on $\overline{\mathbb{D}}_{+}$to conclude that

$$
u_{\Sigma} \geq \operatorname{conv}\left(\left.u_{\Sigma}\right|_{\partial \mathbb{D}}\right)+u_{0}
$$

Since $u_{0}$ is zero on the $y$-axis, we conclude that $u_{\Sigma}=\operatorname{conv}\left(\left.u_{\Sigma}\right|_{\partial \mathbb{D}}\right)$ on the $y$ axis. The other inequality follows from convexity of $u_{\Sigma}$. 


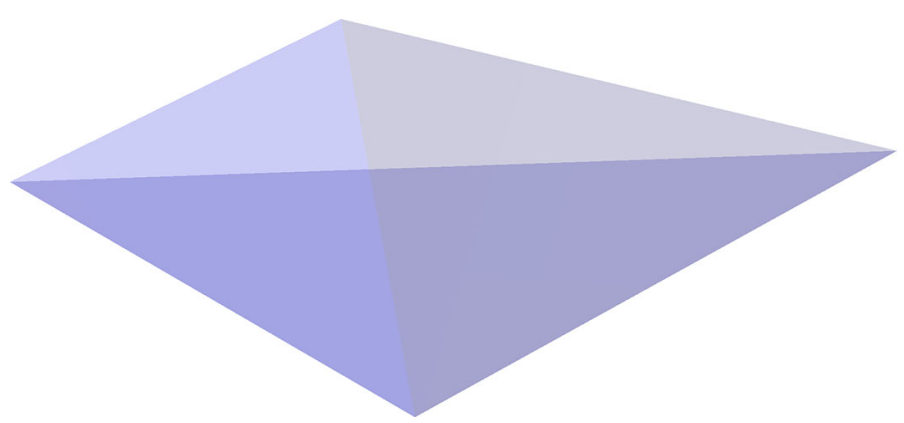

Fig. 5 When $\varphi$ is finite on exactly three points, the domain of dependence $\mathcal{D}_{\varphi}$ is the intersection of the future of three null planes

Remark 4.5 The reason why in the last part of the proof of the previous theorem we did not apply directly Proposition 3.12 to the domain $\mathbb{D}_{+}$is that this would lead to the following inequality $u_{\Sigma} \geq \operatorname{conv}\left(\left.u_{\Sigma}\right|_{\partial \mathbb{D}_{+}}\right)+u_{0}$. However, since the restriction $u_{\Sigma}$ to $\partial \mathbb{D}_{+} \backslash \partial \mathbb{D}$ is not constantly $+\infty$, we can no longer argue that $\operatorname{conv}\left(\left.u_{\Sigma}\right|_{\partial \mathbb{D}_{+}}\right)$coincides with $\operatorname{conv}\left(\left.u_{\Sigma}\right|_{\partial \mathbb{D}}\right)$. So the previous estimate is not useful to control $u_{\Sigma}$ a priori on the $y$-axis.

We then have the following corollary of the results of the previous subsection:

Corollary G. Let $F: \mathbb{H}^{2} \rightarrow \mathbb{H}^{2}$ be a minimal Lagrangian map. Then the image $F\left(\mathbb{H}^{2}\right)$ coincides with the interior of the convex hull of $\overline{F\left(\mathbb{H}^{2}\right)} \cap \partial \mathbb{H}^{2}$.

Proof By Lemma 1.8, $F$ can be realized as the Gauss map of a CGC- $K$ surface $\Sigma$ in $\mathbb{R}^{2,1}$, which is entire. Indeed the first fundamental form coincides with the metric on the source, and is therefore complete. Therefore $\Sigma$ is entire by Remark 1.12. Hence, by applying again Theorem 4.4, the image of the Gauss map of $\Sigma$ (which is identified with $F$ ) coincides with the convex hull of $\overline{F\left(\mathbb{H}^{2}\right)} \cap \partial \mathbb{H}^{2}$.

\section{The case of the ideal triangle}

In this section, we will consider the special case of the regular domain $\mathcal{D}_{\varphi}$ in $\mathbb{R}^{2,1}$, where $\varphi$ takes finite value on precisely three distinct points $\xi_{1}, \xi_{2}, \xi_{3}$ of $\partial \mathbb{D}$, and $\varphi(\xi)=+\infty$ otherwise. This regular domain $\mathcal{D}_{\varphi}$ is the intersection of three half-spaces bounded by null planes. We call such a regular domain a triangular domain (See Fig. 5).

The purpose of this section is to prove the following:

Proposition 5.1 Let $K \in(0,+\infty)$ and let $\varphi: \partial \mathbb{D} \rightarrow \mathbb{R} \cup\{+\infty\}$ be a function with $\varphi\left(\xi_{1}\right), \varphi\left(\xi_{2}\right), \varphi\left(\xi_{3}\right)<+\infty$ and $\varphi(\xi)=+\infty$ otherwise. Then the domain $\mathcal{D}_{\varphi}$ is the domain of dependence of an entire $C G C$ - $K$ surface.

The linear isometry group $\mathrm{SO}_{0}(2,1)$ acts simply transitively on triples of null planes intersecting at the origin. Furthermore, any triple of nonparallel null planes intersect at 
a point. Therefore, up to the action of the affine isometry group of $\mathbb{R}^{2,1}$, all triangular domains are equivalent to each other. Therefore, it is enough to produce a CGC- $K$ surface in any single triangular domain $\mathcal{D}_{\varphi}$. Moreover, using also homethety, it suffices to produce a CGC-1 surface with this property.

\subsection{Harmonic maps to an ideal triangle}

We will construct such a surface $\Sigma$ by using the correspondence between minimal Lagrangian maps and CGC-K surfaces described in Lemma 1.8. In fact, we will construct a minimal Lagrangian map by way of harmonic maps.

From the classical theory of harmonic maps, a harmonic map $f$ from $\mathbb{C}$ to $\mathbb{H}^{2}$ is determined up to isometries of $\mathbb{H}^{2}$ by the Hopf differential $\Phi_{f}$ and the holomorphic energy density $\mathscr{H}_{f}$, which are defined by

$$
\begin{aligned}
& \Phi_{f}=\left(f^{*} h_{\mathbb{H}^{2}}\right)^{2,0}=\langle\partial f, \bar{\partial} f\rangle, \\
& \mathscr{H}_{f}=\|\partial f\|^{2}=\langle\partial f, \partial f\rangle,
\end{aligned}
$$

where we use the decomposition $d f=\partial f+\bar{\partial} f$. It is well-known that $\Phi_{f}$ is a holomorphic quadratic differential. Setting $\mathscr{H}_{f}=e^{2 h}$ and $\Phi_{f}=\phi(\mathbf{z}) d z^{2}$, the function $h$ satisfies the Bochner equation:

$$
\Delta h=e^{2 h}-\frac{|\phi|^{2}}{e^{2 h}} .
$$

Proposition 5.2 [14] Given two hyperbolic surfaces $(S, h)$ and $\left(S^{\prime}, h^{\prime}\right)$, a diffeomorphism $F:(S, h) \rightarrow\left(S^{\prime}, h^{\prime}\right)$ is minimal Lagrangian if and only if there exist harmonic diffeomorphisms $f:\left(S_{0}, X_{0}\right) \rightarrow(S, h)$ and $f^{\prime}:\left(S_{0}, X_{0}\right) \rightarrow\left(S^{\prime}, h^{\prime}\right)$, where $\left(S_{0}, X_{0}\right)$ is a Riemann surface, such that:

(1) $F=f^{\prime} \circ f^{-1}$,

(2) $\Phi_{f}=-\Phi_{f^{\prime}}$, and

(3) $\mathscr{H}_{f}=\mathscr{H}_{f^{\prime}}$.

If there exists a CGC-1 surface $\Sigma$ whose domain of dependence is a triangular domain, then by Theorem $\mathrm{E}$ the image of the Gauss map $G_{\Sigma}: \Sigma \rightarrow \mathbb{H}^{2}$ must be an ideal triangle in $\mathbb{H}^{2}$. From the results of [13], it is known that if a harmonic map $f: \mathbb{C} \rightarrow \mathbb{H}^{2}$ has polynomial Hopf differential of degree $n$, then its image is an ideal polygon with $n+2$ vertices. Hence our strategy is to consider a minimal Lagrangian diffeomorphism $F_{0}=f_{0}^{\prime} \circ f_{0}^{-1}$, where $f_{0}, f_{0}^{\prime}: \mathbb{C} \rightarrow \mathbb{H}^{2}$ are harmonic maps with Hopf differentials

$$
\Phi_{0}=\operatorname{Hopf}\left(f_{0}\right)=-\mathrm{z} d \mathrm{z}^{2} \quad \Phi_{0}^{\prime}=\operatorname{Hopf}\left(f_{0}^{\prime}\right)=\mathrm{z} d \mathrm{z}^{2}
$$

There is a natural choice of solution to the Bochner equation on $\mathbb{C}$ with Hopf differential $\pm \Phi_{0}$, given by the following result: 
Theorem 5.3 [20] Let $\Phi$ be a holomorphic quadratic differential on $\mathbb{C}$ which is not identically zero. Then there exists a unique smooth function $h: \mathbb{C} \rightarrow \mathbb{R}$ which solves

$$
\Delta h=e^{2 h}-|\phi|^{2} e^{-2 h}
$$

such that $e^{2 h}-|\phi|^{2} e^{-2 h}>0$ and the Riemannian metric $e^{2 h}|d z|^{2}$ is complete.

Remark 5.4 Associated to the harmonic map $f_{0}^{\prime}$ is also a constant mean curvature spacelike immersion $\sigma_{H}: \mathbb{C} \rightarrow \mathbb{R}^{2,1}$, which is conformal and also has $f_{0}^{\prime}$ as its Gauss map (see for example [5]). The induced metric on $\sigma_{H}(\mathbb{C})$ is $e^{2 h}|d z|^{2}$. Therefore, the solution $h$ given by Theorem 5.3 has the property that $\sigma_{H}(\mathbb{C})$ is complete, and hence properly embedded. Choosing a normalization so that the mean curvature of $\sigma_{H}$ is $1 / 2$, the CGC-1 immersion $\sigma$ is given by the formula $\sigma(\mathrm{z})=\sigma_{H}(\mathrm{z})-G\left(\sigma_{H}(\mathrm{z})\right)$, where $G$ is the Gauss map of $\sigma_{H}$, taking values in $\mathbb{H}^{2} \subset \mathbb{R}^{2,1}$. This classical observation also holds in Euclidean space. In a future paper, we will use it to derive similar results for constant mean curvature surfaces.

Let $h_{0}$ be the solution of the Bochner equation with Hopf differential $\pm \Phi_{0}$ guaranteed by Theorem 5.3. By Proposition 5.2, this determines up to isometry a minimal Lagrangian diffeomorphism $F_{0}=f_{0}^{\prime} \circ f_{0}^{-1}$, where $f_{0}, f_{0}^{\prime}: \mathbb{C} \rightarrow \mathbb{H}^{2}$. By Lemma 1.8, this in turn determines up to isometry a CGC-1 spacelike immersion $\sigma_{0}: \mathbb{C} \rightarrow \mathbb{R}^{2,1}$. According to the proof of Lemma 1.8, the immersion data (I, $B$ ) of $\sigma_{0}$ are uniquely determined by

$$
\begin{aligned}
\mathrm{I} & =f_{0}^{*} h_{\mathbb{H}^{2}}, \\
\left(f_{0}^{\prime}\right)^{*} h_{\mathbb{H}^{2}}(\cdot, \cdot) & =f_{0}^{*} h_{\mathbb{H}^{2}}(B \cdot, B \cdot),
\end{aligned}
$$

and the condition that $B$ is positive and symmetric for $f_{0}^{*}\left(h_{\mathbb{H}^{2}}\right)$. In the following, we will express I and $B$ explicitly.

\subsection{An expression for the embedding data}

We will ultimately show that the CGC- 1 surface $\sigma_{0}(\mathbb{C})$ is entire; for this, we will need to analyze the asymptotic behavior of $\sigma_{0}$ and in particular of the function $h_{0}$. To this end, it is useful to introduce the local chart

$$
w=(2 / 3) z^{3 / 2}
$$

This means that $w$ is a branch of square root of $z^{3}$, up to the factor $2 / 3$. We remark that $w$ gives a chart on any sector of angle less than $4 \pi / 3$. Since $\Phi_{0}=-z d z^{2}$ has an order 3 rotational symmetry, and the uniqueness part of Theorem 5.3 implies that $h_{0}$ has the same symmetry, the parameter $w$ will be sufficient to understand the whole geometry of the problem.

Remark 5.5 In fact, $h_{0}$ is totally rotationally symmetric since the magniture $|\phi|^{2}$ is the only contribution to the Bochner equation. Even though this remark is not strictly necessary for any of our results, it is worth pointing out that it reduces the construction of $\sigma_{0}$ to the solution of an ordinary differential equation. 
We now give expressions for the embedding data (I, $B)$ of $\sigma_{0}$ in a w coordinate chart. First note that with respect to this coordinate $\Phi_{0}=-d \mathrm{w}^{2}$. Moreover, the logarithmic holomorphic energy density $\tilde{h}_{0}$ with respect to the $w$ chart is related to $h_{0}$ by

$$
\tilde{h}_{0}=h_{0}-\frac{1}{2} \log |z|
$$

and by Eq. (19) with $\phi=1$ it satisfies the Bochner equation $\Delta \tilde{h}_{0}=2 \sinh \left(2 \tilde{h}_{0}\right)$, where the Laplacian is with respect to the metric $|d w|^{2}$.

The first fundamental form is by construction

$$
\mathrm{I}=f_{0}^{*} h_{\mathbb{H}^{2}}=-d \mathrm{w}^{2}+e|d \mathrm{w}|^{2}-d \overline{\mathrm{W}}^{2},
$$

where $e$ is the energy density of $f_{0}$ with respect to the flat metric $|d \mathrm{w}|^{2}$. Then, using the equations $e=\mathscr{H}+\mathscr{L}$, where $\mathscr{L}$ is the anti-holomorphic energy density, and in the w coordinate $\mathscr{H} \mathscr{L}=|\phi|^{2}=1$, we have

$$
e=e^{2 \tilde{h}_{0}}+e^{-2 \tilde{h}_{0}}=2 \cosh \left(2 \tilde{h}_{0}\right),
$$

where $\tilde{h}_{0}$ is the logarithmic holomorphic energy density in the w coordinate. Similarly, the third fundamental form is given by

$$
\mathrm{III}=\left(f_{0}^{\prime}\right)^{*} h_{\mathbb{H}^{2}}=d \mathrm{w}^{2}+2 \cosh \left(2 \tilde{h}_{0}\right)|d \mathrm{w}|^{2}+d \overline{\mathrm{w}}^{2} .
$$

To write $B$ in coordinates, it is helpful to introduce the coordinates $\mathrm{w}=u+i v$, so that $d \mathrm{w}^{2}+d \overline{\mathrm{w}}^{2}=2\left(d u^{2}-d v^{2}\right)$. Then we obtain:

$$
\begin{aligned}
\mathrm{I}=f_{0}^{*} h_{\mathbb{H}^{2}} & =2 \cosh \left(2 \tilde{h}_{0}\right)\left(d u^{2}+d v^{2}\right)-2\left(d u^{2}-d v^{2}\right) \\
& =\left(2 \sinh \left(\tilde{h}_{0}\right)\right)^{2} d u^{2}+\left(2 \cosh \left(\tilde{h}_{0}\right)\right)^{2} d v^{2}
\end{aligned}
$$

and similarly

$$
\begin{aligned}
\mathrm{III}=\left(f_{0}^{\prime}\right)^{*} h_{\mathbb{H}^{2}} & =2 \cosh \left(2 \tilde{h}_{0}\right)\left(d u^{2}+d v^{2}\right)+2\left(d u^{2}-d v^{2}\right) \\
& =\left(2 \cosh \left(\tilde{h}_{0}\right)\right)^{2} d u^{2}+\left(2 \sinh \left(\tilde{h}_{0}\right)\right)^{2} d v^{2} .
\end{aligned}
$$

Now it is easy to see that from Equation (20) that

$$
B=\operatorname{coth}\left(\tilde{h}_{0}\right) d u \otimes \frac{\partial}{\partial u}+\tanh \left(\tilde{h}_{0}\right) d v \otimes \frac{\partial}{\partial v} .
$$

\subsection{A priori estimates for Bochner equation}

In this section we provide the estimates for $\tilde{h}_{0}$ which will allow us to conclude that $\sigma_{0}: \mathbb{C} \rightarrow \mathbb{R}^{2,1}$ is a proper embedding. Let $r=|\mathrm{w}|$ be the radial coordinate with respect to the $w$ chart. A particular case of [13, Lemma 1.2] provides an a priori bound on such rotationally invariant solution. 
Lemma 5.6 There exist constants $C>0$ and $r_{0}>0$ such that

$$
0 \leq \tilde{h}_{0}(w) \leq e^{-C r}
$$

as long as $|w| \geq r_{0}$.

Remark 5.7 Recalling the expression (23) for the shape operator $B$, we see that the principal curvatures of $\sigma_{0}$,

$$
\lambda=\operatorname{coth}\left(\tilde{h}_{0}\right) \quad \mu=\tanh \left(\tilde{h}_{0}\right),
$$

satisfy $\lambda \rightarrow+\infty$ and $\mu \rightarrow 0$ as $|\mathrm{w}| \rightarrow+\infty$.

We will actually need a similar bound, but from below, on the function $\tilde{h}_{0}$, which we prove in the following lemma.

Lemma 5.8 There exist constants $A>0$ and $r_{0}>0$ such that

$$
\tilde{h}_{0}(w) \geq \frac{A}{\sqrt{r}} e^{-2 r},
$$

as long as $|w| \geq r_{0}$.

Proof Recall that for $r>0, \tilde{h}_{0}$ solves the PDE

$$
\Delta \tilde{h}_{0}=2 \sinh \left(2 \tilde{h}_{0}\right)
$$

Now, consider the function

$$
v=v(r)=\frac{A}{\sqrt{r}} e^{-2 r} .
$$

Since $v(r)$ is rotationally symmetric, we have

$$
\Delta v=v^{\prime \prime}(r)+\frac{v^{\prime}(r)}{r}
$$

and by a direct computation we see

$$
\Delta v=\left(4+\frac{1}{4 r^{2}}\right) v .
$$

On the other hand, for fixed $A$, if $r$ is large enough, $v(r)$ is smaller than any power of $1 / r$. Therefore, using the Taylor expansion of the hyperbolic sine near zero, for large enough $r$, independent of $A$ so long as $A<1$ say:

$$
2 \sinh 2 v \sim 4 v+\frac{8}{3} v^{3}<\left(4+\frac{1}{4 r^{2}}\right) v .
$$


Hence there exists $r_{0}$ independent of $A$ so long as $A<1$, such that

$$
\Delta v>2 \sinh 2 v
$$

for every $r \geq r_{0}$.

To conclude, choose $1>A>0$ such that $v\left(r_{0}\right)=A r_{0}^{-1 / 2} e^{-2 r_{0}}<\tilde{h}_{0}(\mathrm{w})$ for all $|\mathrm{w}|=r_{0}$. Then by the maximum principle for this choice of $A$, we have $\tilde{h}_{0} \geq v$ whenever $r \geq r_{0}$. Indeed, $\tilde{h}_{0}-v>0$ on the circle $\left\{r=r_{0}\right\}$ by the choice of $A$, and $\tilde{h}_{0}-v \rightarrow 0$ as $r \rightarrow+\infty$ since each function goes to 0 , so if we suppose that the set $\left\{\tilde{h}_{0}<v\right\} \cap\left\{r \geq r_{0}\right\}$ is non-empty, then $\tilde{h}_{0}-v$ has to assume a negative minimum value. But at the minimum point,

$$
\Delta \tilde{h}_{0}=2 \sinh 2 \tilde{h}_{0}<2 \sinh 2 v<\Delta v,
$$

hence $\Delta\left(\tilde{h}_{0}-v\right)<0$ and this gives a contradiction. Hence we conclude that

$$
\tilde{h}_{0}>v=\frac{A}{\sqrt{r}} e^{-2 r}
$$

for $r \geq r_{0}$ and for a suitable choice of $A>0$, as claimed.

\subsection{Proof of Proposition 5.1}

We begin with several lemmas, which will help us to understand the behavior of the immersion $\sigma_{0}$ in specific directions. The ultimate goal is to show that $\sigma_{0}$ is a proper embedding. First, we argue that since $\sigma_{0}$ is convex, it is at the very least a subset of an entire achronal surface.

Lemma 5.9 Let $\sigma: S \rightarrow \mathbb{R}^{2,1}$ be a $C^{2}$ immersion with everywhere positive definite second fundamental form. Let $G: S \rightarrow \mathbb{H}^{2}$ be the Gauss map of $\sigma$, and suppose that $G$ is injective with convex image. Then $\sigma$ is an embedding and moreover there exists a convex achronal entire surface $\Sigma$ such that the normal of each support plane of $\Sigma$ is in $\overline{G(S)}$, and $\sigma(S)$ is the subset of $\Sigma$ whose support planes have normal contained in $G(S)$.

Proof The strategy of the proof is to construct the support function of $\Sigma$ from the immersion $\sigma$. Let $\pi: \mathbb{H}^{2} \rightarrow \mathbb{D}$ be the radial projection to the disk at height 1 . Define the function $u_{\sigma}: \operatorname{Im}(\pi \circ G) \rightarrow \mathbb{R}$ by

$$
u_{\sigma}(\pi \circ G(\boldsymbol{p}))=\langle\boldsymbol{p},(\pi \circ G(\boldsymbol{p}), 1)\rangle
$$

The fact that the second fundamental form of $\sigma$ is positive definite implies that the function $u_{\sigma}$ is convex, by straightforward calculation of the Hessian of $u_{\sigma}$. Since the domain $\pi \circ G(S)$ of $u_{\sigma}$ is also convex, its convex hull conv $\left(u_{\sigma}\right)$ is equal to $u_{\sigma}$ on $\pi \circ G(S)$ and is equal to $+\infty$ on $\overline{\pi \circ G(S)}^{c}$. By Proposition 2.4, conv $\left(u_{\sigma}\right)$ is dual to an entire achronal surface $\Sigma$. Moreover the Legendre transform gives a homeomorphism 
from the image of $\pi \circ G$ to the subset of $\Sigma$ consisting of points whose support plane has normal contained in the image of $G$. Composing the Legendre transform with $\pi \circ G$, we obtain an embedding $\sigma^{\prime}: S \rightarrow \mathbb{R}^{2,1}$. By construction, the Gauss map of $\sigma^{\prime}$ is equal to $G$ and the support function of $\sigma^{\prime}$ is equal to $u_{\sigma}$ (compare Eq. (7)).

Since $\sigma$ is an immersion with positive definite second fundamental form, it is locally the graph of a convex function. By the local nature of the formula to recover a surface from its support function (Eq. (6)), the immersions $\sigma$ and $\sigma^{\prime}$ must agree. Therefore $\sigma$ is an embedding and its image is exactly those points of $\Sigma$ whose support plane has normal in $G(S)$.

We will take advantage of the global symmetries of the CGC surface $\sigma_{0}(\mathbb{C})$ and its achronal extension $\Sigma$ as in Lemma 5.9.

Lemma 5.10 There exists a dihedral group $\Gamma<\operatorname{Isom}\left(\mathbb{R}^{2,1}\right)$ of order 6 which leaves the surface $\Sigma$ invariant. Moreover, $\Gamma$ is generated by a linear elliptic isometry in $\mathrm{SO}_{0}(2,1)$ of order 3 , and by a reflection in a timelike plane.

Proof By Theorem 1.1, the surface $\sigma_{0}(\mathbb{C})$ is determined up to a global isometry by the embedding data, namely the first fundamental form I of Equation (21) and the shape operator $B$ of Equation (23).

Since the solution $h_{0}$ to Bochner equation is rotationally invariant, and the holomorphic quadratic differentials $\pm z d z^{2}$ have an order 3 rotational symmetry, the embedding data (I, $B$ ) have a dihedral group of (intrinsic) isometries generated by the rotation $\alpha: \mathbf{z} \mapsto \omega z$ (where $\omega$ is a cubic root of the identity) and by the conjugation $\beta: \mathbf{z} \mapsto \bar{z}$. By the uniqueness part of Theorem 1.1, the embeddings $\sigma_{0}$ and $\sigma_{0} \circ \alpha$ differ by a global isometry $A \in \operatorname{Isom}\left(\mathbb{R}^{2,1}\right)$. Such $A$ must necessarily preserve orientation and time-orientation, and fix the point $\sigma_{0}(0)$ and the normal vector $N\left(\sigma_{0}(0)\right)$. Hence $A \in \mathrm{SO}_{0}(2,1)$ is a rotation, and has order three by a similar argument.

Analogously, one shows that $\sigma_{0}$ and $\sigma_{0} \circ \beta$ differ by a time-orientation preserving and orientation-reversing isometry $B$ which fixes the geodesic $\sigma_{0}(\{\operatorname{Im}(z)=0\})$ of $\sigma_{0}(\mathbb{C})$ pointwise. We have thus obtained a representation of the dihedral group $\langle\alpha, \beta\rangle$ of order 6 in $\operatorname{Isom}\left(\mathbb{R}^{2,1}\right)$, whose image leaves $\sigma_{0}(\mathbb{C})$ invariant.

Since Lemma 5.9 defines $\Sigma$ canonically in terms of the embedding $\sigma_{0}$, it is also invariant under the same group of isometries.

Finally, we show that the surface $\sigma_{0}(\mathbb{C})$ looks like a properly embedded surface along its planes of symmetry. First, the following general lemma characterizes properly embedded spacelike curves contained in a timelike plane in terms of their speed and curvature.

Lemma 5.11 Let $\gamma:[0,+\infty) \rightarrow \mathbb{R}^{1,1}$ be a spacelike curve with curvature $\kappa:$ $[0,+\infty) \rightarrow \mathbb{R}_{+}$and speed $v:[0,+\infty) \rightarrow \mathbb{R}_{+}$. Assume that

$$
\int_{0}^{\infty} \exp \left(\int_{0}^{r} \kappa(s) v(s) d s\right) v(r) d r=+\infty .
$$

Then $\gamma$ is proper. 
Proof Let $T:[0,+\infty) \rightarrow \mathbb{R}^{1,1}$ be the unit tangent vector field along $\gamma$ and $N$ be the normal vector. Denote by $t=t(r)$ the arclength, so that

$$
\frac{d t}{d r}=v(r)
$$

We have that $\langle T, T\rangle=1,\langle N, N\rangle=-1$, and $\langle T, N\rangle=0$. Moreover,

$$
\left\{\begin{array}{l}
\frac{d T}{d t}=\kappa N \\
\frac{d N}{d t}=\kappa T
\end{array}\right.
$$

Observe that the lightlike directions of $T+N$ and $T-N$ are fixed. Let $\xi_{-}$be a futurepointing lightlike vector parallel to $T-N$, and consider the function $\rho=\left\langle\gamma, \xi_{-}\right\rangle$. As $\frac{d \gamma}{d t}=T$, we have

$$
\left\{\begin{array}{l}
\frac{d \rho}{d t}=\left\langle T, \xi_{-}\right\rangle \\
\frac{d^{2} \rho}{d t}=\left\langle\kappa N, \xi_{-}\right\rangle=\kappa\left\langle T, \xi_{-}\right\rangle=\kappa \frac{d \rho}{d t}
\end{array}\right.
$$

where we have used that $\left\langle T-N, \xi_{-}\right\rangle=0$. So $\frac{d \rho}{d t}(t)=\frac{d \rho}{d t}(0) \exp \left(\int_{0}^{t} \kappa(r(\tau)) d \tau\right)$ or in terms of the parameter $r$,

$$
\frac{d \rho}{d t}(t(r))=C \exp \left(\int_{0}^{r} \kappa(s) v(s) d s\right) .
$$

So

$$
\frac{d \rho}{d r}(r)=\frac{d \rho}{d t}(t(r)) \cdot v(r)=C \exp \left(\int_{0}^{r} \kappa(s) v(s) d s\right) v(r)
$$

and by the assumption,

$$
\int_{0}^{+\infty} \frac{d \rho}{d r} d r=+\infty
$$

Therefore $\left\langle\gamma(r), \xi_{-}\right\rangle \rightarrow+\infty$ and this implies that $\gamma$ is proper.

We now apply this general result to the intersection of $\sigma_{0}(\mathbb{C})$ with its planes of symmetry. One of these three identical curves is the fixed points of the conjugation $\beta: \mathbf{z} \mapsto \overline{\mathbf{z}}$.

Corollary 5.12 The restriction of $\sigma_{0}(z)$ to the real axis in the $z$ coordinate is a proper spacelike curve.

Proof Since this curve is fixed by the conjugation symmetry $\beta$, it must be contained in the timelike plane fixed by the corresponding reflection of $\mathbb{R}^{2,1}$. Identify this timelike plane with $\mathbb{R}^{1,1}$. We check properness at each end in turn. For each case, we choose 
a branch $w=\frac{2}{3} z^{3 / 2}$ as above. The negative real axis in the $z$ chart corresponds to the imaginary axis $\mathrm{w}=0+i v$ in the $\mathrm{w}$ chart. By Eq. (21) we see that the metric on this ray is $\left(2 \cosh \left(\tilde{h}_{0}\right)\right)^{2} d v^{2}$ which always larger than $4 d v^{2}$, and so this end is complete. Since it is contained in a timelike plane, completeness implies that it is properly embedded.

The harder case is the positive real axis, which corresponds to the real axis $\mathrm{w}=$ $u+0 i$ in the w chart. Here the induced metric is $\left(2 \sinh \left(\tilde{h}_{0}\right)\right)^{2} d u^{2}$, which tends to 0 as $u \rightarrow \infty$. With respect to the $u$ coordinate, Equations (21) and (23) show that the velocity is $v=2 \sinh \left(\tilde{h}_{0}(u)\right)$ and the curvature is $\kappa=\operatorname{coth}\left(\tilde{h}_{0}(u)\right)$. Therefore

$$
\exp \left(\int_{0}^{u} \kappa(s) v(s) d s\right)=\exp \left(\int_{0}^{u} 2 \cosh \left(\tilde{h}_{0}(s)\right) d s\right) \geq \exp (2 u)
$$

and using $\sinh \tilde{h}_{0} \geq \tilde{h}_{0}$ together with the estimate of Lemma 5.8,

$$
\int_{u_{0}}^{\infty} \exp \left(\int_{0}^{u} \kappa(s) v(s) d s\right) v(u) d u \geq \int_{u_{0}}^{\infty} \exp (2 u)\left(\frac{A}{\sqrt{u}} \exp (-2 u)\right) \rightarrow+\infty \text {. }
$$

Hence we conclude from Lemma 5.11 that this end of the curve is also proper.

Now we conclude the proof of the main result of this section.

Proof of Proposition 5.1 Let $\sigma_{0}$ be the CGC-1 immersion constructed in the previous section, which is actually an embedding by Lemma 5.9, and let $\Sigma$ be the convex achronal entire surface constructed from $\sigma_{0}$ via Lemma 5.9. We will show that the image $\sigma_{0}(\mathbb{C}) \subseteq \Sigma$ coincides with $\Sigma$, and this will show that the image of $\sigma_{0}$ is entire.

Since the image of $\sigma_{0}$ is open and nonempty in $\Sigma$, it suffices to show that its boundary is empty. For this purpose, let us assume there exists a sequence $z_{n} \in \mathbb{C}$ such that $\sigma_{0}\left(z_{n}\right) \rightarrow \boldsymbol{p} \in \partial \sigma_{0}(\mathbb{C})$. As the image of the Gauss map $f_{0}^{\prime}: \mathbb{C} \rightarrow \mathbb{H}^{2}$ of $\sigma_{0}$ is an ideal triangle, we can extract a subsequence ( still denoted $z_{n}$ ) such that $f_{0}^{\prime}\left(z_{n}\right)$ converge either to an ideal vertex or to a point on an edge of the ideal triangle. We will rule out both possibilities and thus get a contradiction.

Let us first suppose that $f_{0}^{\prime}\left(z_{n}\right)$ converge to an ideal vertex. Since support planes converge to support planes, it follows that $\Sigma$ admits a lightlike support plane $P$ (as a limit of the spacelike support planes to $\sigma_{0}(\mathbb{C})$ at $\sigma_{0}\left(\mathbf{z}_{n}\right)$ ), which must be parallel to one of the three null directions in the closure of the Gauss map image. Now using Lemma 5.10, let $Q$ be the timelike plane of symmetry of $\Sigma$ such that reflection in $Q$ leaves $P$ invariant.

By Corollary 5.12, the intersection of $\sigma_{0}(\mathbb{C})$ with $Q$ is a proper spacelike curve. In particular, $\Sigma$ can contain no point in $Q \cap P$. In particular, the point $\boldsymbol{p}$ is not in $Q$. Now let $\boldsymbol{p}^{\prime} \in \Sigma$ be the reflection of $\boldsymbol{p}$ across $Q$. Since the reflection leaves $P$ invariant, $P$ must also be the support plane of $\Sigma$ at $\boldsymbol{p}^{\prime}$. Hence the midpoint of $\boldsymbol{p}$ and $\boldsymbol{p}^{\prime}$ lies on the plane $Q$ and still has null support plane $P$, which contradicts the fact that the intersection of $\sigma_{0}(\mathbb{C})$ with $Q$ is a proper spacelike curve

We are thus left with the case that $f_{0}^{\prime}\left(z_{n}\right)$ converge to an a point of an edge of the ideal triangle which is the image of the Gauss map. Let us now consider the new immersion $\sigma_{1}: \mathbb{C} \rightarrow \mathbb{R}^{2,1}$ defined by: 


$$
\sigma_{1}(\mathbf{z})=\sigma_{0}(\mathbf{z})+f_{0}^{\prime}(\mathbf{z})
$$

where we are considering $f_{0}^{\prime}$ as a map valued in $\mathbb{H}^{2} \subset \mathbb{R}^{2,1}$. (Since $f_{0}^{\prime}=\boldsymbol{n}$ is the Gauss map of $\sigma_{0}$, the immersion $\sigma_{1}$ is the normal evolution of $\sigma_{0}$ at time 1.) By a direct computation, one obtains

$$
d \sigma_{1}(v)=d \sigma_{0}(v)+D_{v} \boldsymbol{n}=d \sigma_{0}((\mathbb{1}+B)(v)),
$$

where $B$ is the shape operator of $\sigma_{0}$, and therefore the first fundamental form of $\sigma_{1}$ equals:

$$
\left.\left\langle d \sigma_{1}(v), d \sigma_{1}(w)\right\rangle=\mathrm{I}(\mathbb{1}+B) v,(\mathbb{1}+B) w\right) .
$$

By a direct computation from Eqs. (21), (22) and (23), this metric has the form $4 e^{2 \tilde{h}_{0}}|d \mathrm{w}|^{2}$ which is a complete metric on $\mathbb{C}$ as $\tilde{h}_{0}>0$ (see Lemma 5.6). Therefore $\sigma_{1}(\mathbb{C})$ is a proper immersion (Remark 1.12). Now, since $\sigma_{0}\left(\mathbf{z}_{n}\right)$ is converging to $\boldsymbol{p}$, and $f_{0}^{\prime}\left(z_{n}\right)$ is converging to some interior point of $\mathbb{H}^{2}$, the sequence $\sigma_{1}\left(\mathbf{z}_{n}\right)$ is converging in $\mathbb{R}^{2,1}$ by Eq. (25). But the sequence $z_{n}$ escapes from compact sets of $\mathbb{C}$, hence $\sigma_{1}\left(z_{n}\right)$ is diverging in $\mathbb{R}^{2,1}$, and this gives a contradiction.

In conclusion, following Lemma 5.9, $\Sigma=\sigma_{0}(\mathbb{C})$, so in particular $\sigma_{0}(\mathbb{C})$ is entire. Then by Corollary 1.17 its domain of dependence must be the intersection of the futures of a set of null planes. Since the image of the Gauss map is an ideal triangle, Theorem E implies that this set must contain exactly three null planes. Since all triangular regular domains are equivalent up to isometry of $\mathbb{R}^{2,1}$, this concludes the proof.

Remark 5.13 It actually turns out, as mentioned in Remark 5.4, that $\sigma_{1}$ is a constant mean curvature embedding. The completeness of the first fundamental form of $\sigma_{1}$ is therefore also a consequence of more general results. However, the existence of such constant mean curvature surface only allows us to prove the second part of Proposition 5.1, namely, to show that there is no converging sequence $\sigma_{0}\left(\mathrm{z}_{n}\right)$ for which the Gauss map converge to an interior point of $\mathbb{H}^{2}$. Tools from CMC surfaces are not helpful to tackle the first point, that is, excluding the existence of lightlike support planes for $\sigma_{0}(\mathbb{C})$.

The reason why such strategy fails is that, starting from an entire CMC surface, one can follow the normal flow in the past to obtain a CGC immersion, but it is hard to prove that it is entire (in fact, it will not be complete in general). Hence we do not adopt the language of CMC surfaces here, and the technical estimates on the holomorphic energy, leading to Corollary 5.12, are the essential ingredient for our proof.

In order to apply the entire CGC- $K$ surfaces provided by Proposition 5.1 as barriers for the general case, we will need to translate Proposition 5.1 in terms of MongeAmpère equations. In fact, fix three distinct points $\xi_{1}, \xi_{2}, \xi_{3}$ and three values $v_{1}, v_{2}, v_{3}$.

Let $\Sigma^{K}$ be the surface provided by Proposition 5.1 with $\varphi\left(\xi_{i}\right)=v_{i}$. Then the support function $u_{\Sigma^{K}}$ satisfies the Monge-Ampère equation (9). Moreover, by Theorem 4.4, $u_{\Sigma^{K}}$ is necessarily affine on each side of the triangle $T$ having vertices $\xi_{1}, \xi_{2}, \xi_{3}$. Finally, the Legendre transform of $u_{\Sigma^{K}}$ gives the surface $\Sigma^{K}$, since $u_{\Sigma^{K}}$ is convex and lower semicontinuous. 
Hence we can reformulate our result in terms of Monge-Ampère equations:

Corollary 5.14 Given three distinct points $\xi_{1}, \xi_{2}, \xi_{3} \in \partial \mathbb{D}$, let $T$ be the triangle in $\overline{\mathbb{D}}$ with vertices $\xi_{1}, \xi_{2}, \xi_{3}$. For any affine function $l: \mathbb{D} \rightarrow \mathbb{R}$, there exists a solution $u$ to the problem

$$
\left\{\begin{array}{l}
\operatorname{det} D^{2} u(z)=\frac{1}{K}\left(1-|z|^{2}\right)^{-2} \text { for every } z \in \operatorname{int}(T) \\
\left.u\right|_{\partial T}=\left.l\right|_{\partial T}
\end{array}\right.
$$

such that the graph of the Legendre transformation of $u$ is an entire surface.

\section{Existence and uniqueness for the Minkowski problem}

In this section we will prove the main result (Theorem F) concerning the Minkowski problem, namely the existence and uniqueness of entire surfaces of prescribed Gaussian curvature in any regular domain $\mathcal{D}_{\varphi}$, where $\varphi$ is finite on at least three points of $\partial \mathbb{D}$.

\subsection{Statement of the Monge-Ampère problem}

We start by constructing solutions to the Monge-Ampère equation (8). Recall that the convex envelope of a function $\varphi: \partial \mathbb{D} \rightarrow \mathbb{R} \cup\{+\infty\}$, introduced in Definition 2.6, is:

$$
\operatorname{conv}(\varphi)(z)=\sup \left\{f(z) \mid f: \overline{\mathbb{D}} \rightarrow \mathbb{R} \text { is affine, }\left.f\right|_{\partial \mathbb{D}} \leq \varphi\right\} \text {. }
$$

Moreover, we denote by $\Omega_{\varphi} \subseteq \mathbb{D}$ the interior of the convex hull of $\{\xi \in \partial \mathbb{D} \mid \varphi(\xi)<$ $+\infty\}$.

Theorem 6.1 Let $\varphi: \partial \mathbb{D} \rightarrow \mathbb{R} \cup\{+\infty\}$ be any lower semi-continuous function which is finite on at least three distinct points. Let $\psi \in C^{\infty}\left(\Omega_{\varphi}\right)$ such that $a<\psi(z)<b$ for some $a, b>0$ and for every $z \in \Omega_{\varphi}$. Then there exists a unique closed convex function $u: \overline{\mathbb{D}} \rightarrow \mathbb{R} \cup\{+\infty\}$ which is a solution to

$$
\begin{cases}\operatorname{det} D^{2} u(z)=\frac{1}{\psi(z)}\left(1-|z|^{2}\right)^{-2} & \text { for } z \in \Omega_{\varphi} \\ u(z)=\operatorname{conv}(\varphi)(z) & \text { for } z \in \overline{\mathbb{D}} \backslash \Omega_{\varphi} .\end{cases}
$$

Moreover, $u$ is smooth on $\Omega_{\varphi}$ and gradient-surjective.

Recall by Proposition 2.7 that $\operatorname{conv}(\varphi)$ is equal to $\varphi$ on $\partial \mathbb{D}$ and on any chord $\left[\xi_{1}, \xi_{2}\right]$ of $\partial \Omega_{\varphi}$ it is the unique affine function interpolating $\varphi\left(\xi_{1}\right)$ and $\varphi\left(\xi_{2}\right)$.

\subsection{Proof of existence}

We will split the proof of Theorem 6.1 in two parts, by proving first the existence and then the uniqueness. 
Proof of the existence part of Theorem 6.1 Let us split the proof into several steps.

Step 1: To simplify notation, we will write $h=\operatorname{conv}(\varphi)$. Let us first construct an approximating sequence $u_{n}$. Let $\Omega_{n}$ be an exhaustion of $\Omega_{\varphi}$ by strictly convex domains with smooth boundary, satisfying $\Omega_{n} \subset \subset \Omega_{\varphi}$ and $\Omega_{n} \subset \subset \Omega_{n+1}$ for every $n$. By the classical theory of Monge-Ampère equations (Theorem 3.7), there exists a solution $u_{n}: \Omega_{n} \rightarrow \mathbb{R}$ of the problem

$$
\left\{\begin{array}{l}
\operatorname{det} D^{2} u_{n}(\mathrm{z})=\frac{1}{\psi(\mathrm{z})}\left(1-|\mathrm{z}|^{2}\right)^{-2} \text { for every } \mathrm{z} \in \Omega_{n} \\
\left.u_{n}\right|_{\partial \Omega_{n}}=\left.h\right|_{\partial \Omega_{n}}
\end{array}\right.
$$

which is continuous in $\bar{\Omega}_{n}$. By Theorem $3.9, u_{n}$ is strictly convex and therefore smooth by Theorem 3.8.

Step 2: We now need to prove some uniform a priori estimates on the $u_{n}$. We claim that:

$$
h(\mathrm{z})-\frac{1}{\sqrt{a}} \sqrt{1-|\mathrm{z}|^{2}} \leq u_{n}(\mathrm{z}) \leq h(\mathrm{z})
$$

for every $z \in \Omega_{n}$. Indeed, the inequality $u_{n} \leq h$ follows from the fact that $h$ is the convex envelope of $\varphi$ and $u_{n}$ is convex. For the other inequality, for every linear function $l$ on the disk, the comparison principle (Corollary 3.5) gives

$$
l(\mathbf{z})-\frac{1}{\sqrt{a}} \sqrt{1-|\mathbf{z}|^{2}} \leq u_{n}(\mathbf{z}) \text {. }
$$

Taking the supremum over all linear functions less than or equal to $\varphi$ (compare with the proof of Proposition 3.12), we conclude

$$
h(\mathrm{z})-\frac{1}{\sqrt{a}} \sqrt{1-|\mathrm{z}|^{2}} \leq u_{n}(\mathrm{z})
$$

Step 3: We can now produce the solution $u_{\infty}$ as a limit of the $u_{n}$. In fact, it follows from the previous step that the functions $u_{n}$ are uniformly bounded on $\Omega_{n_{0}}$ for $n \geq n_{0}$. Moreover, since the $u_{n}$ are convex, by a classical argument they are equicontinuous on $\Omega_{n_{0}}$ for $n \geq n_{0}+1$, where the coefficient of equicontinuity depends on the uniform bound on $\Omega_{n_{0}+1}$ and on the distance between $\Omega_{n_{0}}$ and $\Omega_{n_{0}+1}$.

Thus by the Ascoli-Arzelà theorem and a standard diagonal argument, we can extract a subsequence which converges uniformly on compact sets of $\Omega_{\varphi}$ to a function $u_{\infty}: \Omega_{\varphi} \rightarrow \mathbb{R}$. By Lemma $3.3, u_{\infty}$ satisfies

$$
\operatorname{det} D^{2} u_{\infty}(\mathrm{z})=\frac{1}{\psi(\mathrm{z})}\left(1-|\mathrm{z}|^{2}\right)^{-2}
$$

Hence we have again that $u_{\infty}$ is strictly convex (Theorem 3.9) and therefore smooth (Theorem 3.8).

Step 4: Now define the closed convex function $u$ by $u=\operatorname{conv}\left(u_{\infty}\right)$. As $u_{\infty}$ is already convex, we have that $u$ coincides with $u_{\infty}$ on $\Omega_{\varphi}$. It remains to show that $u=h$ 
on $\overline{\mathbb{D}} \backslash \Omega_{\varphi}$. Both are infinite away from $\bar{\Omega}_{\varphi}$, so we restrict attention to $\partial \Omega_{\varphi}$. Let us first show that $u=h$ on $\bar{\Omega}_{\varphi} \cap \partial \mathbb{D}$. Using (27), one obtains

$$
h(\mathrm{z})-\frac{1}{\sqrt{a}} \sqrt{1-|\mathrm{z}|^{2}} \leq u_{\infty}(\mathrm{z}) \leq h(\mathrm{z}) .
$$

Taking the convex hull preserves these inequalities. Since $\frac{1}{\sqrt{a}} \sqrt{1-|z|^{2}}$ vanishes on $\partial \mathbb{D}$, we conclude immediately that $u=h=\varphi$ on $\partial \mathbb{D}$.

Step 5: We are left with showing $u=h$ on $\partial \Omega_{\varphi} \cap \mathbb{D}$. Let $c=\left[\xi_{1}, \xi_{2}\right]$ be any chord in $\partial \Omega_{\varphi}$. Let $D_{c}$ be the half-plane in $\mathbb{D}$ bounded by $\left[\xi_{1}, \xi_{2}\right]$ which intersects $\Omega_{\varphi}$ and let $u_{c}^{a}$ be the unique solution of the problem

$$
\left\{\begin{array}{l}
\operatorname{det} D^{2} u_{c}^{a}(\mathrm{z})=\frac{1}{\sqrt{a}}\left(1-|z|^{2}\right)^{-2} \quad \text { for every } z \in D_{c} \\
\left.u_{c}^{a}\right|_{\partial D_{c}}=0
\end{array} .\right.
$$

If $c$ is the geodesic $\{x=0\}$ and $D_{c}=\mathbb{D}_{+}=\{x \geq 0\}$, then such solution was provided explicitly in Equation (17), as it is the support function of a surface of revolution $\Sigma_{0}^{a}$, and by inspection it is continuous in $\overline{\mathbb{D}}_{+}$. In general, $u_{c}^{a}$ is the support function of a surface obtained by applying a linear isometry in $\operatorname{SO}_{0}(2,1)$ to $\Sigma_{0}^{a}$. Therefore also the solution $u_{c}^{a}$ is continuous on $\bar{D}_{c}$.

By an argument similar to above (Equation (27)) we get

$$
h(\mathrm{z})+u_{c}^{a}(\mathrm{z}) \leq u_{n}(\mathrm{z}) \leq h(\mathrm{z}) .
$$

By passing to the limit, we thus obtain

$$
h(\mathrm{z})+u_{c}^{a}(\mathrm{z}) \leq u_{\infty}(\mathrm{z}) \leq h(\mathrm{z})
$$

Taking convex envelopes as above and using that $u_{c}^{a}$ vanishes on $c$, we conclude that $u=h$ on $c$.

\subsection{Proof of uniqueness}

Let us now complete the proof by showing the uniqueness of the solution $u$.

Proof of the uniqueness part of Theorem 6.1 We split the proof into two steps.

Step 1: Let $u$ now be any solution of (26). As above, set $h=\operatorname{conv}(\varphi)$. First we show that $u$ must satisfy the inequalities:

$$
h(\mathrm{z})-\frac{1}{\sqrt{a}} \sqrt{1-|\mathrm{z}|^{2}} \leq u(\mathrm{z}) \leq h(\mathrm{z})
$$

and, for every chord $c$ in $\partial \Omega_{\varphi}$,

$$
h(\mathrm{z})+u_{c}^{a}(\mathrm{z}) \leq u(\mathrm{z})
$$


where $u_{c}^{a}$ is the solution, defined on the half-plane $D_{c}$ bounded by $c$, of:

$$
\left\{\begin{array}{l}
\operatorname{det} D^{2} u_{c}^{a}(\mathbf{z})=\frac{1}{a}\left(1-|\mathbf{z}|^{2}\right)^{-2} \quad \text { for every } \mathbf{z} \in D_{c} \\
\left.u_{c}^{a}\right|_{\partial D_{c}}=0
\end{array} .\right.
$$

The inequality $u \leq h$ is obvious. The other inequality follows from an adaptation of the argument in [4, Proposition 3.9], where more details can be found. First, up to composing with an isometry, suppose $0 \in \mathbb{D}$ is in $\Omega_{\varphi}$. Fix $r \in(0,1)$ and let $u_{r}: \bar{\Omega}_{\varphi} \rightarrow \mathbb{R}$ be defined by $u_{r}(\mathrm{z})=u(r \mathrm{z})$. It is then easy to check that

$$
\operatorname{det} D^{2} u_{r}(\mathrm{z}) \leq \frac{1}{a}\left(1-|z|^{2}\right)^{-2}
$$

Let us now define

$$
h_{r}=\operatorname{conv}\left(\left.u_{r}\right|_{\partial \Omega_{\varphi}}\right)
$$

Since $u_{r}$ is continuous up to the boundary of $\Omega_{\varphi}$ and $\left.\left(u_{r}\right)\right|_{\partial \Omega_{\varphi}}=\left.\left(h_{r}\right)\right|_{\partial \Omega_{\varphi}}$, again by the comparison principle we get

$$
h_{r}(\mathrm{z})+v(\mathrm{z}) \leq u_{r}(\mathrm{z})
$$

where $v \leq 0$ can be any of the functions $(1 / \sqrt{a}) \sqrt{1-|z|^{2}}$ or $u_{c}^{a}$, for every chord $c$.

It then turns out that

$$
h(\xi) \leq \liminf _{r \rightarrow 1} h_{r}(\xi)
$$

for every $\xi \in \partial \Omega_{\varphi}$. In order to show this, let $f$ be an affine function on $\mathbb{D}$ such that $f<h$. Since $u$ is lower-semicontinuous, the sublevel set $\{z \in \overline{\mathbb{D}}: u(z) \leq f(z)\}$ is compact. Since $f$ is finite everywhere, it is contained in $\Omega_{\varphi}$ and since it is compact it is contained in $r_{0} \Omega_{\varphi}$ for some $r_{0}<1$.

This shows that

$$
u_{r}(\xi)=u(r \xi)>f(r \xi)=: f_{r}(\xi)
$$

for every $\xi \in \partial \Omega_{\varphi}$ and every $r \geq r_{0}$. That is, $\left.\left(f_{r}\right)\right|_{\partial \Omega_{\varphi}}<\left.\left(u_{r}\right)\right|_{\partial \Omega_{\varphi}}$, which implies $f_{r} \leq h_{r}$. Taking the limit as $r \rightarrow 1$, this implies

$$
f(\mathbf{z}) \leq \liminf _{r \rightarrow 1} h_{r}(\mathbf{z})
$$

The inequality (33) then follows, as $h(\xi)$ is defined as the supremum of $f(\xi)$ over all such affine functions $f$. Finally, taking the limit as $r \rightarrow 1$ in (32), we conclude the proof of (30) and (31). 
Step 2: Let $u_{1}$ and $u_{2}$ be any two solutions of (26). Using the inequality (30), we obtain

$$
-\frac{1}{\sqrt{a}} \sqrt{1-|z|^{2}} \leq u_{1}(\mathrm{z})-u_{2}(\mathrm{z}) \leq \frac{1}{\sqrt{a}} \sqrt{1-|\mathrm{z}|^{2}}
$$

and similarly from (31),

$$
u_{c}^{a}(\mathrm{z}) \leq u_{1}(\mathrm{z})-u_{2}(\mathrm{z}) \leq-u_{c}^{a}(\mathrm{z})
$$

As already pointed out, $u_{c}^{a}$ is continuous up to $c$, on which it is zero. This implies that $u_{1}-u_{2}$ extends continuously to zero on $\partial \Omega_{\varphi}$ and therefore by the comparison principle of Theorem 3.4, we have $\min \left(u_{1}-u_{2}\right)=0$. By reversing the roles of $u_{1}$ and $u_{2}$, we have $\min \left(u_{2}-u_{1}\right)=0$ and thus $u_{1}=u_{2}$.

\subsection{Proof of entireness}

In this subsection, we prove that the solution $u$ obtained in Theorem 6.1 is the support function of an entire surface, which is equivalent to the statement that $u$ is gradient surjective. This is the key step to conclude the existence of entire surfaces solving the Minkowski problem, hence in particular the classification of entire CGC- $K$ surfaces in $\mathbb{R}^{2,1}$.

Proof of the entireness part of Theorem 6.1 The graph of the convex dual of $u$ is an achronal surface $\Sigma$ as in Lemma 5.9. As long as no point of $\Sigma$ has a support plane whose slope lies outside $\Omega_{\varphi}$, we can recover all of $\Sigma$ as the graph of the Legendre transform of $u$ on $\Omega_{\varphi}$, and so $\Sigma$ must be the spacelike CGC surface that we are looking for.

Suppose for the sake of contradiction that $\Sigma$ contains a point $\boldsymbol{p}$ at which it admits a support plane $P$ whose slope lies outside of $\Omega_{\varphi}$. By convex duality, the plane $P$ must be one of the planes in the boundary of $\Omega_{\varphi}$ at which $u$ is finite:

$$
P=\left\{\boldsymbol{x} \in \mathbb{R}^{2,1}:\langle\boldsymbol{x},(\mathrm{z}, 1)\rangle=u(\mathrm{z})\right\}
$$

for some $\xi \in \partial \Omega_{\varphi}$. If $z$ is on the boundary of $\mathbb{D}$, set $\xi_{1}=z$ and choose two other points $\xi_{2}, \xi_{3}$ such that $\varphi\left(\xi_{i}\right)<+\infty$. Note that this is possible by the assumption that $\varphi$ is finite at at least 3 points. If $z$ lies on a chord $\left[\xi_{1}, \xi_{2}\right]$ of $\partial \Omega_{\varphi}$, choose one other point $\xi_{3}$ at which $\varphi\left(\xi_{3}\right)<+\infty$. Let us call $T_{0}$ the ideal triangle with vertices $\xi_{1}, \xi_{2}, \xi_{3}$.

Let $l$ be the unique affine function with the property that $l\left(\xi_{i}\right)=\varphi\left(\xi_{i}\right)$ for $i=$ 1, 2, 3. By Proposition 5.1, there exists an entire $K$-surface $\Sigma_{0}$, for $K=b$, with support function $u_{0}: \overline{\mathbb{D}} \rightarrow \mathbb{R} \cup\{+\infty\}$ satisfying $\left.u_{0}\right|_{\partial T_{0}}=\left.l\right|_{\partial T_{0}}$ and $u_{0}(\mathrm{z})=+\infty$ for $z \in \overline{\mathbb{D}} \backslash \overline{T_{0}}$.

Now, the functions $u$ and $u_{0}$ satisfy:

$$
\operatorname{det} D^{2} u=\frac{1}{\psi(z)}\left(1-|z|^{2}\right)^{-2} \leq \frac{1}{b}\left(1-|z|^{2}\right)^{-2}=\operatorname{det} D^{2} u_{0} .
$$


Moreover $u\left(\xi_{i}\right)=u_{0}\left(\xi_{i}\right)$ for $i=1,2,3$. Since $T_{0}$ is a polygon, the restriction of $u$ to $T_{0}$ is continuous by [10, Theorem 2]. Since $u$ is convex, we have $\left.u\right|_{\partial T_{0}} \leq\left. u_{0}\right|_{\partial T_{0}}$. Hence by the comparison principle (Corollary 3.5), $\left.u\right|_{T_{0}} \leq\left. u_{0}\right|_{T_{0}}$. This shows that $\Sigma$ is contained in the future of $\Sigma_{0}$. But the surface $\Sigma_{0}$ is entire and hence completely in the future of the support plane $P$ defined in Equation (34). This contradicts the assumption $p \in \Sigma \cap P$.

\subsection{Conclusion of Minkowski and CGC problem}

We can now apply all the proved results and state the main theorems concerning the Minkowski problem and the CGC problem. In fact, in Theorem 6.1 we construct a solution of the problem

$$
\begin{cases}\operatorname{det} D^{2} u(\mathrm{z})=\frac{1}{\psi(\mathrm{z})}\left(1-|\mathrm{z}|^{2}\right)^{-2} & \text { for } \mathrm{z} \in \Omega_{\varphi} \\ u(\mathrm{z})=\operatorname{conv}(\varphi)(\mathrm{z}) & \text { for } \mathrm{z} \in \overline{\mathbb{D}} \backslash \Omega_{\varphi} .\end{cases}
$$

for every lower semicontinuous function $\varphi$ finite on at least three points and every smooth bounded function $\psi$ defined on $\Omega_{\varphi}$. Using this result, we now derive the complete solution to the Minkowski and CGC problems for entire spacelike surfaces.

Theorem $\mathrm{F}$. Given any regular domain $\mathcal{D}$ in $\mathbb{R}^{2,1}$ which is not a wedge and any smooth function $\psi$ defined on the image of the generalized Gauss map of $\partial \mathcal{D}$ such that a $<$ $\psi<b$ for some $a, b>0$, there exists a unique entire spacelike surface $\Sigma$ in $\mathcal{D}$ whose domain of dependence is $\mathcal{D}$ and whose curvature function satisfies:

$$
\kappa(\boldsymbol{p})=\psi \circ G_{\Sigma}(\boldsymbol{p})
$$

for every $\boldsymbol{p} \in \Sigma$, where $G_{\Sigma}$ is the Gauss map of $\Sigma$.

Proof of Theorem $F$ The existence part follows from Theorem 6.1. Indeed, by Proposition 2.5 the regular domain $\mathcal{D}$ must be equal to $\mathcal{D}_{\varphi}$ for some closed function $\varphi$ which is finite at at least 3 points. Then Theorem 6.1 produces a function $u$ whose Legendre transform has the required properties.

Uniqueness is a straightforward consequence of Theorem 6.1 together with Theorem 4.4. Indeed, by Theorem 4.4 , for any surface $\Sigma$ satisfying the condition of Theorem $F$, the image of its Gauss map projected to the Klein model $\mathbb{D}$ of $\mathbb{H}^{2}$, must be $\Omega_{\varphi}$, i.e. the interior of the convex hull of $\{\xi \in \partial \mathbb{D}: \varphi(\xi)<+\infty\}$. Moreover, the two bullet points of Theorem 4.4 imply that its support function $u_{\Sigma}$ agrees with $\operatorname{conv}(\varphi)$ on $\overline{\mathbb{D}} \backslash \Omega_{\varphi}$. Therefore, $u_{\Sigma}$ must be a solution of the problem (26) of Theorem 6.1, and by the uniqueness part of Theorem 6.1 , we conclude that $\Sigma$ is unique.

As a particular case, we therefore obtain the solution to the CGC problem in regular domains different from a wedge:

Theorem A. Fix $K>0$. Given any regular domain $\mathcal{D} \subset \mathbb{R}^{2,1}$ which is not a wedge, there exists a unique entire CGC K-surface whose domain of dependence is $\mathcal{D}$. 
We conclude with the following classification result for entire CGC- $K$ surfaces.

Corollary C. Fix $K>0$. There is a bijection between the set of future-convex entire surfaces of constant Gaussian curvature $K$ in $\mathbb{R}^{2,1}$ and the set of lower semicontinuous functions $\varphi: \partial \mathbb{D} \rightarrow \mathbb{R} \cup\{+\infty\}$ finite on at least three points, which is defined by $\left.\Sigma \mapsto\left(u_{\Sigma}\right)\right|_{\partial \mathbb{D}}$.

Proof Let $\Sigma$ be a future-convex entire CGC- $K$ surface. By Corollary 1.17, the domain of dependence of $\Sigma$ is of the form $\mathcal{D}_{\varphi}$ for some lower semi-continuous function $\varphi$. In light of Theorem A, it remains only to rule out the possibility that $\varphi$ is finite at 0,1 , or 2 points.

But by Theorem E, the image of the Gauss map of $\Sigma$ must be the interior of the convex hull of those points where $\varphi$ is finite. Clearly the image of the Gauss map of $\Sigma$ must be nonempty, which rules out the cases of 0 or 1 points, and by strict convexity it must also have interior, which rules out the case of 2 points.

As a remark, we mention that there is a natural action of $\operatorname{Isom}\left(\mathbb{R}^{2,1}\right)=\operatorname{SO}_{0}(2,1) \rtimes$ $\mathbb{R}^{2,1}$ on the set of future-convex entire surfaces of constant Gaussian curvature. Under the bijection of Corollary $\mathrm{C}$, this action corresponds to a natural action of the semidirect product $\mathrm{SO}_{0}(2,1) \rtimes \mathbb{R}^{2,1}$ on the set of lower semi-continuous functions. See also [17].

\section{Foliations by CGC surfaces}

The purpose of this section is to show that any regular domain $\mathcal{D}$ which is not a wedge if foliated by the (unique) CGC- $K$ surfaces $\Sigma_{K}$ having domain of dependence $\mathcal{D}$, as for Theorem A.

Theorem D. For every regular domain $\mathcal{D}$ in $\mathbb{R}^{2,1}$ which is not a wedge, there exists a unique foliation by properly embedded $C G C$ - $K$ surfaces, as $K \in(0, \infty)$.

Proof There are three main steps in the proof. The first two steps will show that the $K$-surfaces provide a foliation of a region of $\mathcal{D}$. Then the third step will show that this region "fills up" $\mathcal{D}$ close to $\partial \mathcal{D}$ and close to infinity.

Step 1: Let us first show that the CGC- $K$ surfaces are disjoint: more precisely, if $K_{1}<K_{2}$, then $\Sigma_{K_{1}}$ is in the future of $\Sigma_{K_{2}}$. Let $u_{1}$ and $u_{2}$ be the support functions of $\Sigma_{K_{1}}$ and $\Sigma_{K_{2}}$ respectively. Both $u_{1}$ and $u_{2}$ satisfy the inequalities (30) and (31) for $K=K_{1}$. Therefore, their difference tends to zero at the boundary of $\Omega_{\varphi}$. By the comparison principle, $u_{2}-u_{1}$ cannot have an interior minimum, and so it follows that $u_{2}>u_{1}$ strictly on the whole domain $\Omega_{\varphi}$. From the formula for the convex dual, we see immediately that $\Sigma_{K_{1}}$ lies weakly in the future of $\Sigma_{K_{2}}$; since both surfaces are entire, the strict inequality $u_{2}>u_{1}$ implies that they cannot be tangent at any point, and so we in fact have that $\Sigma_{K_{1}}$ lies strictly in the future of $\Sigma_{K_{2}}$.

Step 2: It remains to show that, for every point $x \in \mathcal{D}$, there exists a CGC surface $\Sigma_{K}$ such that $x \in \Sigma_{K}$. Hence let

$$
\mathcal{I}_{-}:=\left\{K \in(0, \infty): x \in \overline{I^{-}\left(\Sigma_{K}\right)}\right\}
$$


and analogously

$$
\mathcal{I}_{+}:=\left\{K \in(0, \infty): x \in \overline{I^{+}\left(\Sigma_{K}\right)}\right\} .
$$

We emphasize that $\mathcal{I}_{-}$corresponds to surfaces lying above $\boldsymbol{x}$ and $\mathcal{I}_{+}$corresponds to surfaces lying below $\boldsymbol{x}$. In this step we show that $\mathcal{I}_{-}$and $\mathcal{I}_{+}$are nonempty.

Let $\varphi=\varphi_{\mathcal{D}}$. The point $\boldsymbol{x}$ corresponds to an affine plane $P_{\boldsymbol{x}}$ in $\mathbb{D} \times \mathbb{R}$ which lies strictly below the graph of $\varphi$. Moreover, the point $x$ is in $I^{+}\left(\Sigma_{K}\right)$ if and only if the plane $P_{x}$ lies below the graph of the support function $u_{\Sigma_{K}}$. By (30) we have for all K

$$
\operatorname{conv}(\varphi)(\mathrm{z})-\frac{1}{\sqrt{K}} \sqrt{1-|\mathrm{z}|^{2}} \leq u_{\Sigma_{K}}(\mathrm{z}) \leq \operatorname{conv}(\varphi)(\mathrm{z})
$$

Therefore, as $K \rightarrow \infty$, the support function $u_{\Sigma_{K}}$ converges to $\operatorname{conv}(\varphi)$, so for $K$ large enough, $P_{x}$ lies below the graph of $u_{\Sigma_{K}}$. This shows that $\mathcal{I}_{+}$is nonempty.

On the other hand, let $\mathcal{D}_{0}$ be any triangular domain containing $\mathcal{D}$. Since it is invariant under rescaling, $\mathcal{D}_{0}$ is foliated by rescaled copies of its corresponding CGC-1 triangular surface $\Sigma^{0}$. As the rescaled surfaces tend towards infinity, their curvature tends to zero. In particular, for some $K$ small enough, $\Sigma_{K}^{0}$ is in the future of $x$. By the same application of the comparison principle as in the proof of entireness (Sect. 6.4), $\Sigma_{K}$ lies in the future of $\Sigma_{K}^{0}$. This shows that $\mathcal{I}_{-}$is nonempty.

Step 3: By the previous two steps, $\mathcal{I}_{-}$and $\mathcal{I}_{+}$are nonempty and connected, with $\inf \mathcal{I}_{-}=0$ and $\sup \mathcal{I}_{+}=+\infty$. In this step, we show that $\sup \mathcal{I}_{-}=\inf \mathcal{I}_{+}=: K_{x}$ and $x \in \Sigma_{K_{x}}$.

Let $K_{-}=\sup \mathcal{I}_{-}$, and let $K_{i} \in \mathcal{I}_{-}$be an increasing sequence tending to $K_{-}$. Then $\Sigma_{K_{i}}$ are a decreasing sequence of surfaces with $\boldsymbol{x}$ in their past. The corresponding support functions $u_{i}$ form an increasing sequence bounded from above by $\operatorname{conv}(\varphi)$, so they converge uniformly on compact subsets of $\Omega_{\varphi}$ to a limit $u_{\infty}$. By Lemma 3.3, $u_{\infty}$ is a solution of the Monge-Ampère equation (9) on $\Omega_{\varphi}$ with curvature $K_{-}$. Passing to the limit, inequalities (30) and (31) applied to $u_{i}$ show that $u_{\infty}$ is equal to $\operatorname{conv}(\varphi)$ on $\partial \Omega_{\varphi}$. Hence by the uniqueness part of Theorem 6.1, $u_{\infty}$ is the support function of $\Sigma_{K_{-}}$.

Since the functions $u_{i}$ converge uniformly on compact sets to a strictly convex limit, the convergence must be at least $C^{1}$, and therefore the dual surface $\Sigma_{K_{i}}$ also converge locally uniformly to $\Sigma_{K_{-}}$. In particular, since $x \in I^{-}\left(\Sigma_{K_{i}}\right)$, it follows that $x \in \overline{I^{-}\left(\Sigma_{K_{-}}\right)}$. Therefore $K_{-} \in \mathcal{I}_{-}$.

Similarly, if $K_{+}=\inf \mathcal{I}_{+}$, we can produce an increasing family of surfaces $\Sigma_{K_{i}}$, with a corresponding decreasing family of support functions $u_{i}$. Since $\boldsymbol{x}$ is in the future of each surface $\Sigma_{K_{i}}$, the corresponding affine plane $P_{x}$ in $\mathbb{D} \times \mathbb{R}$ lies below the graph of each function $u_{i}$. Since the $u_{i}$ are bounded below, they again converge uniformly on compact sets and as above the limit is the support function of $\Sigma_{K_{+}}$. We conclude similarly that $K_{+} \in \mathcal{I}_{+}$.

We have now shown that $\mathcal{I}_{-}$and $\mathcal{I}_{+}$are closed as subsets of $(0, \infty)$. They clearly cover the interval $(0, \infty)$, so they must have nonempty intersection. On the other 
hand, the intersection consists precisely of those $K$ such that $x \in \Sigma_{K}$, which by

Step 1 must be a single value. Hence $K_{-}=K_{+}$, and this surface contains $\boldsymbol{x}$.

\section{Open questions}

We conclude by mentioning an open question on the subject. Theorem A provides a classification of entire CGC- $K$ surfaces in $\mathbb{R}^{2,1}$, which is also stated in Corollary C. It would be interesting to classify complete CGC- $K$ surfaces (which are automatically entire), in terms of the function $\varphi=\left.\left(u_{\Sigma}\right)\right|_{\partial \mathbb{D}}$. This would give a classification of all $C^{2}$ isometric immersion of the hyperbolic plane into $\mathbb{R}^{2,1}$.

In [4] a characterization of surfaces with bounded principal curvature was obtained. That is, an entire surface $\Sigma$ has bounded principal curvatures (that is, the principal curvatures are in an interval $[1 / C, C]$ for some $C>0$ ) if and only if $\varphi$ has the Zygmund regularity. Let us observe that, if $\Sigma$ has bounded principal curvatures, then it is complete, since the Gauss map is bi-Lipschitz in this case. In particular, this characterization does not depend on $K$.

Hence the class of complete CGC- $K$ surfaces correspond to a subset of the space of lower semicontinuous functions, finite on at least three points, which contains Zygmund regular functions. In particular, it contains Lipschitz functions. We actually have some negative examples: first, the entire CGC- $K$ surfaces considered in Sect. 5, are not complete, since the induced metric is homothetic to an ideal triangle in $\mathbb{H}^{2}$. In this case, the support function is only finite on three points. Moreover, in [4] another example was provided, namely an entire non-complete surface (the induced metric is isometric to the universal cover of $\mathbb{H}^{2} \backslash\{p\}$ for a point $p \in \mathbb{H}^{2}$ ), whose support function on $\partial \mathbb{D}$ is:

$$
\varphi(\xi)=\left\{\begin{array}{ll}
a & \text { if } \xi=\xi_{0} \\
b & \text { if } \xi \neq \xi_{0}
\end{array},\right.
$$

for any $a<b$.

We remark that in all example we know of noncomplete entire CGC- $K$ surfaces, the support function $\varphi$ on $\partial \mathbb{D}$ has the property that there is a point $\xi_{0} \in \partial \mathbb{D}$ at which

$$
\liminf _{\substack{\xi \rightarrow \xi_{0} \\ \xi \neq \xi_{0}}} \varphi(\xi)>\varphi\left(\xi_{0}\right) .
$$

It would be interesting to know if this is a necessary or sufficient condition.

Acknowledgements We thank Jean-Marc Schlenker for his interest and encouragement throughout. The third author also wishes to thank Shing-Tung Yau for inspiring interest in the problem.

Open Access This article is distributed under the terms of the Creative Commons Attribution 4.0 International License (http://creativecommons.org/licenses/by/4.0/), which permits unrestricted use, distribution, and reproduction in any medium, provided you give appropriate credit to the original author(s) and the source, provide a link to the Creative Commons license, and indicate if changes were made. 


\section{References}

1. Barbot, T., Béguin, F., Zeghib, A.: Prescribing Gauss curvature of surfaces in 3-dimensional spacetimes: application to the Minkowski problem in the Minkowski space. Ann. Inst. Fourier (Grenoble) 61(2), 511-591 (2011)

2. Bonsante, F., Fillastre, F.: The equivariant Minkowski problem in Minkowski space. Ann. Inst. Fourier (Grenoble) 67(3), 1035-1113 (2017)

3. Bonsante, F.: Flat spacetimes with compact hyperbolic Cauchy surfaces. J. Differ. Geom. 69(3), 441521 (2005)

4. Bonsante, F., Seppi, A.: Spacelike convex surfaces with prescribed curvature in (2+1)-Minkowski space. Adv. Math. 304, 434-493 (2017)

5. Choi, H.I., Treibergs, A.: Gauss maps of spacelike constant mean curvature hypersurfaces of Minkowski space. J. Differ. Geom. 32(3), 775-817 (1990)

6. Danciger, J.: A geometric transition from hyperbolic to anti-de Sitter geometry. Geom. Topol. 17(5), 3077-3134 (2013)

7. do Carmo, M.P.: Differential Geometry of Curves and Surfaces. Prentice-Hall Inc., Englewood Cliffs (translated from the Portuguese) (1976)

8. Fillastre, F., Seppi, A.: Spherical, hyperbolic and other projective geometries: convexity, duality, transitions. In: Papadopoulos A (ed) Sixteen essays on non-Euclidean geometry. European Mathematical Society Publishing House, Helsinki (2018)

9. Guan, B., Jian, H.-Y., Schoen, R.M.: Entire spacelike hypersurfaces of prescribed Gauss curvature in Minkowski space. J. Reine Angew. Math. 595, 167-188 (2006)

10. Gale, D., Klee, V., Rockafellar, R.T.: Convex functions on convex polytopes. Proc. Am. Math. Soc. 19, 867-873 (1968)

11. Gutiérrez, C.E.: The Monge-Ampère Equation. Progress in Nonlinear Differential Equations and their Applications, vol. 44. Birkhäuser, Boston (2001)

12. Hano, J., Nomizu, K.: On isometric immersions of the hyperbolic plane into the Lorentz-Minkowski space and the Monge-Ampère equation of a certain type. Math. Ann. 262(2), 245-253 (1983)

13. Han, Z.-C., Tam, L.-F., Treibergs, A., Wan, T.: Harmonic maps from the complex plane into surfaces with nonpositive curvature. Comm. Anal. Geom. 3(1-2), 85-114 (1995)

14. Labourie, F.: Surfaces convexes dans l'espace hyperbolique et $\mathbf{C P}^{1}$-structures. J. Lond. Math. Soc. (2) 45(3), 549-565 (1992)

15. Li, A.-M.: Spacelike hypersurfaces with constant Gauss-Kronecker curvature in the Minkowski space. Arch. Math. 64, 534-551 (1995)

16. Rockafellar, R.T.: Convex Analysis. Princeton Mathematical Series, No. 28. Princeton University Press, Princeton (1970)

17. Seppi, A.: Spacelike surfaces of constant Gaussian curvature in Lorentz-Minkowski space. In: Camino, R.L. (ed.) Differential Geometry in Lorentz-Minkowski space, pp. 143-167. EUG, Granada (2017)

18. Smith, G.: Constant scalar curvature hypersurfaces in (3+1)-dimensional GHMC Minkowski spacetimes. J. Geom. Phys. 128, 99-117 (2018)

19. Trudinger, N., Wang, X.-J.: The Monge-Ampére equation and its geometric applications. In: Handbook of Geometric Analysis, vol. 7 of Adv. Lect. Math. (ALM), pp 467-524. Int. Press, Somerville (2008)

20. Wan, T.Y.-H., Au, T.K.-K.: Parabolic constant mean curvature spacelike surfaces. Proc. Am. Math. Soc. 120(2), 559-564 (1994)

Publisher's Note Springer Nature remains neutral with regard to jurisdictional claims in published maps and institutional affiliations. 